\title{
2PC*: a distributed transaction concurrency control protocol of multi-microservice based on cloud computing platform
}

\author{
Pan Fan', Jing Liu' ${ }^{1 *}$ Wei Yin², Hui Wang ${ }^{2}$, Xiaohong Chen ${ }^{1}$ and Haiying Sun ${ }^{1}$
}

\begin{abstract}
The two-phase commit (2PC) protocol is a key technique for achieving distributed transactions in storage systems such as relational databases and distributed databases. 2PC is a strongly consistent and centralized atomic commit protocol that ensures the serialization of the transaction execution order. However, it does not scale well to large and high-throughput systems, especially for applications with many transactional conflicts, such as microservices and cloud computing. Therefore, 2PC has a performance bottleneck for distributed transaction control across multiple microservices. In this paper, we propose $2 \mathrm{PC}^{*}$, a novel concurrency control protocol for distributed transactions that outperforms 2PC, allowing greater concurrency across multiple microservices. 2PC* can greatly reduce overhead because locks are held throughout the transaction process. Moreover, we improve the faulttolerance mechanism of $2 \mathrm{PC}^{*}$ using transaction compensation. We also implement a middleware solution for transactions in microservice support using $2 \mathrm{PC}^{*}$. We compare $2 \mathrm{PC}^{*}$ to $2 \mathrm{PC}$ by applying both to Ctrip MSECP, and $2 \mathrm{PC}^{*}$ outperforms $2 \mathrm{PC}$ in workloads with varying degrees of contention. When the contention becomes high, the experimental results show that $2 \mathrm{PC}^{*}$ achieves at most a 3.3x improvement in throughput and a $67 \%$ reduction in latency, which proves that our scheme can easily support distributed transactions with multi-microservice modules. Finally, we embed our middleware scheme in the PaaS cloud platform and demonstrate its strong applicability to cloud computing through long-term analysis of the monitoring results in the cloud platform.
\end{abstract}

Keywords: 2PC, Distributed transactions, Microservices, Protocol optimization, Cloud computing, PaaS platform

\section{Introduction}

With the rapid and iterative development of Internet products, the traditional monolithic-service architecture is not suitable for the current large-scale data business scenarios. Therefore, microservice architecture has gradually replaced the traditio-nal approach and has become an important topic in industrial and academic circles. Microservice architecture [1] is widely used in large-scale cloud computing platforms and applica-tion development. The key to microservice architecture is to provide flexibility for program development and the reuse of fine-grained services.

\footnotetext{
* Correspondence: jliu@sei.ecnu.edu.cn

${ }^{1}$ East China Normal University, Shanghai 200062, China

Full list of author information is available at the end of the article
}

Microservices can be developed by different-domain teams to support business applications, and they can be implemented in various languages and access multiple underlying databases. In a distributed system, we usually deploy various microservice modules, which are homogeneous or heterogeneous systems composed of service clusters. Meanwhile, most microservices are used in cross-service and cross-resource scenarios. In other words, they need to access multiple databases in different environments when processing business. When an application invokes multiple microservices, distributed transactions are needed to support the consistent updating of these underlying databases and to ensure data consistency throughout the system. In recent years, academia and industry have carried out 
conside-rable amounts of research on the data consistency of certain distributed databases, such as Spanner [2] and OceanBase (https://oceanbase.alipay. $\mathrm{com} /$ ). Fortunately, microservices are closely related to these distri-buted databases in transactions, although supporting data-consistent distributed transactions across multi-microservice modules is even more challenging research [3].

The traditional technique is to implement a distributed transaction using the two-phase commit (2PC) protocol [4]. Unfortunately, this does not work well in large-scale and high-throughput systems, especially for applications with a large number of transaction conflicts [5]. The reason is that locks are held throughout the 2PC process. However, the number of modules across microservices is large, and it is necessary to support users with extremely high numbers of concurrent requests. For this reason, due to the limitations of the $2 \mathrm{PC}$ protocol, the performance of the original business will be seriously reduced, possibly rendering it unusable. Other approaches include persistent message queue patterns for loosely coupled distributed transactions [6,7], which require additional application logic to compensate for failed transaction steps, thus increasing the cost of the system and possibly affecting the experience of users. In many business scenarios, such as e-commerce and e-finance, to ensure that the entire system always has strong data consistency, it must be controlled with the strictest consistency protocols, e.g., 2PC. Although systems such as YugaByte [8] and Found-ationDB [9] can support distributed transactions for a single database, this is not yet scalable in distributed systems with multi-microservice modules.

In view of the above problems, we aim to find a solution suitable for consistently distributed transactions in the micro-service architecture. On the one hand, it is strictly required to meet the basic principles of data consistency in the hetero-geneous distributed system of the microservice architecture. On the other hand, the solution must achieve the same level of throughput as the original microservice business. In other words, it is expected to obtain a high processing performance under the scenario of an extremely high number of concurrent user requests.

In the paper, we propose $2 \mathrm{PC}^{*}$, which is a novel distributed transaction control protocol that can extract more concurrent processing capabilities under highintensity competitive wor-kloads than previous approaches for a multi-microservice. $2 \mathrm{PC}^{*}$ is an optimized protocol based on the traditional 2PC. It utilizes a twolevel asynchronous lock to reduce the over-head of synchronous blocking caused by a surge in the num-ber of transactions, thereby avoiding deadlocks. To achieve this, we propose a novel optimistic lock, i.e., the SAOL. Additionally, $2 \mathrm{PC}^{*}$ uses a runtime protocol for transaction concurrency control to reduce the probability of conflicts between transactions.

In a distributed system environment where microservices are located, especially in cloud computing platforms, there are uncontrollable factors in service, e.g., service loss and network delays. Moreover, microservices are often called remotely through a gateway, such as XML-RPC or SOAP (simple object access protocol), which increases the proba-bility of these conditions occurring. Therefore, we improve the transaction compensation mechanism to achieve the ulti-mate consistency of distributed transactions across micro-services.

Finally, we implement a middleware solution for transa-ctions distributed across microservices based on $2 \mathrm{PC}^{*}$ and deploy it on a specific PaaS cloud platform. Specifically, we use the Netty framework [10] to complete RPCs (remote procedure calls) [11] between transaction roles. Netty is based on Java NIO (nonblocking input and output), so its I/O operation is asynchronous and non-blocking. Therefore, the throughput and stability of RPCs can be greatly improved. We adapt our middleware to two popular microservice frameworks, Spring Cloud and Dubbo [12, 13]. We implement $2 \mathrm{PC}^{*}$ and evaluate its performance using a case of the MSECP platform. 2PC* outperforms the original 2PC in workloads with varying degrees of contention. When the contention is high, $2 \mathrm{PC} \%$ s $\mathrm{s}$ throughput is nearly 10 times that of 2PC. As the system scales across microservices and cont-ention increases, the throughput of $2 \mathrm{PC}^{*}$ continues to grow, while the $2 \mathrm{PC}$ throughput drops to almost zero with no capacity to scale.

The rest of this paper is organized as follows. Section "Overview" elaborates on the overview of our scheme and the classic approach. In Section "Design", we introduce the design details of the $2 \mathrm{PC}$, including the design of SAOL and a runtime protocol. The implementation of the middleware solution based on $2 \mathrm{PC}^{*}$ will be introduced in Section "Implementation". In Section "Evaluation", we will give the experimental data of the middleware solution implemented with $2 \mathrm{PC}$ * and compare it with 2PC. Section "Related Work" discusses some related work. Finally, Section "Conclusion" concludes this paper.

\section{Overview \\ Low performance traditional approaches $2 P C$ and $O C C$}

In consistent transactional processing for traditional distributed databases, developers prefer the trans-action's strongest isolation level, such as serializability [14], to simplify the correctness criteria for concurrent transactions. Therefore, to ensure strict serializability, traditional distrib-uted storage systems usually run standard transactional concurrency control schemes, such as the 
OCC (optimistic concurrency control) [15] combines with $2 \mathrm{PC}$.

Unfortunately, 2PC and OCC perform poorly under com-petitive workloads in large-scale conflict transactions. We introduce a case that simulates the process of a customer buying two items from a store (Table 1). The process contains two fragments, $F_{1}$ and $F_{2}$, each of which reduces the stock quantity of different items. Each fragment can be executed atomically on the server where it is located. However, in the entire distributed system, an additional distributed transac-tion control protocol is needed to prevent fragmented transa-ctions across servers from being non-serializable and interla-ced. For example, suppose the store keeps the stock quantities of item $_{1}$ and item $_{2}$ unchanged and always sells the two items in a bundle. In the absence of a distributed transaction control protocol, the user can purchase item $m_{1}$ but not item $_{2}$, while another user can purchase item ${ }_{2}$ but not item .

We evaluate the performance of $2 \mathrm{PC}$ combined with OCC with two transactions, $T_{1}$ and $T_{2}$. Both purchase the same item $_{1}$ and item $_{2}$ stored on different services. When OCC detection is performed during the execution of $2 \mathrm{PC}$, any interleaving of $T_{1}$ and $T_{2}$ will cause the process to abort. For example, if $T_{2}$ reads the stock number after $T_{1}$ reads it but before $T_{1}$ commits its update to item $1, T_{2}$ will not be able to verify the process and abort later. We introduce another example where both $T_{1}$ and $T_{2}$ are aborted during $2 \mathrm{PC}$ because their corresponding $2 \mathrm{PC}$ precommit instructions are processed by the service in different orders.

However, the performance of $2 \mathrm{PC}$ combined with OCC under high-intensity workloads is far from satisfactory, especially across high-concurrency microservices. 2PC acqu-ires locks on data access for each transaction. When threads perform a conflicting operation, they serialize the transac-tions' execution order. In the example described above, once $T_{1}$ modifies the stock

Table $1 \mathrm{~A}$ fragment of new-order transaction containing two pieces

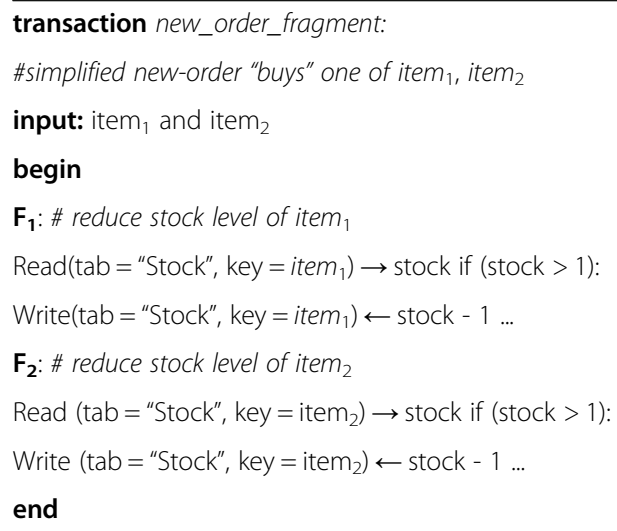

quantity of item $_{1}, T_{2}$ must be blocked until $T_{1}$ completes its entire process and commits successfully. In addition to blocking, 2PC also prevents deadlock by pass-ive thread abort [2]. However, as the number of competing threads increases, so does the probability of deadlocks. In addition, effective deadlock prevention mechanisms [16] (e.g., wound-wait) have many false positives. As a result, even without a real deadlock, most threads will still be abort-ed unexpectedly.

\section{New distributed Transaction's characteristics in microservice architecture}

ACID [17] is a design concept for transactions in traditional databases to ensure the correctness of data and avoid errors such as Read-Committed and RepeatableRead. However, in distributed systems, especially at the application level, it is more important to meet business requirements than to pursue strict system characteristics. According to the CAP principle, strong consistency $(\mathrm{C})$, availability $(\mathrm{A})$, and partition tolera-nce $(\mathrm{P})$ [18] cannot be met simultaneously. However, BASE theory adopts a completely different design idea than ACID. BASE sacrifices strong consistency for high availability and eventual consistency [19], which can be achieved through appropriate methods and is consistent with the characteristics of distributed systems in reality. On this basis, distributed transactions are mostly focused on the application layer for microservices. Therefore, it is necessary to not only ensure the data's eventual consistency but also obtain high availab-ility in the system.

\section{PC* optimize lock in two-phase commit}

According to the details described below, our optimized transmission control protocol 2PC* avoids lock-blocking du-ring the two-phase commit between transactions, especially under scenarios with high-level contention.

$2 \mathrm{PC}^{*}$ optimizes the inefficient synchronizationblocking lock in 2PC and replaces it with a novel secondary asyn-chronous optimistic lock (SAOL). Borrowing from the design of the MVCC (multi-version concurrency control) [20], the SAOL allocates an ever-growing sequence of ver-sions to each transaction step, which is similar to the snapshot. The fine granularity of locks can be broken down into two levels of optimistic locks, borrowing from OCC (optimistic concurrency control) [15]. The SAOL allows multiple transactions to perform updates to the same transaction frag-ment concurrently, with two specific snapshots controlling the order in which transactions are executed, i.e., begin-Version and commitVersion. Meanwhile, the SAOL adopts a two-level optimistic lock (i.e., one composed of a firstLock and secondLock) to control the transaction commit. Using the 
BASE mechanism, the firstLock is responsible for controlling the resources in the main process between multiple transactions; thus, the secondLock can separate from it and compensate for the unfinished process in the firstLock.

\section{PC* simplifies conflicts to commit}

$2 \mathrm{PC}^{*}$ is able to change and optimize the order of execution for transactions because it uses two phases of indicators to commit. In the beginning phase, when multimicroservice modules participate in the same distributed transaction, 2PC* does not immediately execute the subsequent process but instead adds the order relations (i.e., conflicts) between trans-actions to the neighbourList of the directed graph, which can be denoted as GraphNode. We conduct a preliminary merge and de-duplication of conflicts for the neighbourList. In the commit phase, GraphNode then combines all the conflict information and distributes it to all the microservices. $2 \mathrm{PC}^{*}$ then further simplifies the transaction conflicts for the neighbourList so that it performs better under highcompetition transaction scenarios, such as those involving microservice architecture.

\section{A middleware based on cloud platform for distributed transaction control with $2 \mathrm{PC}^{*}$}

Based on $2 \mathrm{PC}^{*}$, we implement a middleware scheme that supports consistent distributed transactions for microservices. Our prototype contains over 23,000 lines of Java code, 17000 of which are for transaction concurrency control, and is based on the Spring-Boot framework. In particular, we adopt the Netty framework to complete the underlying communication between transaction roles. We deploy it in a specific PaaS cloud platform. Experimental data prove that our scheme has very good performance for multi-microservice scenarios with high concurrent requests from users, including higher throu-ghput and lower latency.

\section{Design}

The design of the $2 \mathrm{PC}^{*}$ protocol includes a novel optimistic lock mechanism (i.e., the SAOL), a concurrency control prot-ocol for transactions and a compensation measure.

In this section, we first explain the design of the SAOL. Then, we introduce a concurrency control protocol in $2 \mathrm{PC}^{*}$, an optimizing strategy, and a verification of its correctness. Finally, we provide a fault-tolerant mechanism.

\section{Secondary asynchronous optimistic-lock}

The novel 2PC"'s SAOL replaces the synchronous blocking lock with a high-performance secondary asynchronous lock. The process in each transaction fragment is identified by a unique version number, i.e., snapshot. We adopt Twitter's distributed identification number generation algorithm Snow-flake [21], which contains a timestamp to identify the execu-tion order of the transaction fragment. The specific design of the SAOL is described below.

\section{Transaction property initialization}

In the SAOL, we first define four key attribute fields in the transaction object, which are represented as follows.

- value: This indicates the current actual value of the transaction object.

- beginVersion: That is the version sequence number at the beginning of the process.

- commitVersion: That is the version at the time the transaction was committed.

- lock: This is responsible for locking up the resources of uncommitted transactions. The lock is divided into two different levels, namely the firstLock and the secondLock, which represent the two phases of lock respectively. And we specify that in the secondLock, it needs to contain firstLock information.

\section{Begin transaction process}

First, we need obtain the begin-Version in the transaction object $T_{1}$, denoted $b_{-} v$, and then determine whether there is a lock in $T_{1}$. If no lock exists, we try to obtain the latest committed transaction object directly from the version number interval $\left[0, b_{-} v\right]$ and obtain the current latest value through its beginVersion. Otherwise, the subsequent process is executed according to the following three branches.

- Case 1. If there is another transaction object $T_{2}$ committing a transaction, then the value of $T_{2}$ is locked. At this point, we need to wait for $T_{2}$ to finish committing transactions, then poll and retry to obtain the current latest value.

- Case 2. In case 1 , if the waiting time of $T_{1}$ has passed a certain threshold, denoted WAIT_TIME_OUT, but $T_{2}$ 's value is still locked, it can be determined that $T 2$ is facing some unexpected exceptions, e.g., a network delay or downtime. We can simply assume that $T_{2}$ has been interrupted and it can release lock directly.

- Case 3. $T_{2}$ 's lock may have been remained because it has not been released properly. In this case, $T_{2}$ 's transaction was committed and its firstLock released successfully, but some unforeseen exceptions occurred in the secondLock, so the transaction could not be committed, thus causing the lock to remain. 


\section{Transaction pre-commit process}

At this point, $T_{1}$ has completely obtained its latest value, so we can perform the $T_{1}$ first-phase commit, denoted preCommit. This process can be divided into three branches as follows.

- Case 1. For any two transaction objects $T_{1}$ and $T_{2}$, in the version sequence interval $\left(b_{-} v,+\infty\right)$ corresponding to their values, we determine whether another transaction object $T_{x}$ is updating the data. If this condition matches, it means that $T_{x}$ might have changed the value. At this point, both $T_{1}$ and $T_{2}$ need to roll back their transactions directly, and then we can complete the process.

- Case 2. Determine whether a lock exists in both $T_{1}$ and $T_{2}$, that is, whether their transaction resources are locked. If so, $T_{1}$ and $T 2$ need to directly roll back transactions and we can terminate the process directly.

- Case 3. If neither of the above two cases matches, we set the firstLock of $T_{1}$ and $T_{2}$ to locked, write the latest data to value at this time and commit the transaction.

\section{Transaction second-commit process}

First, we determine whether the firstLock of $T_{1}$ or $T_{2}$ is locked; if so, we commit the transaction directly. The secondLock that belongs to $T_{1}$ and $T_{2}$, it can be completely separated from the main process, and we can then use an asynchronous mode of the thread to release the secondLock and commit transactions in the second phase. Therefore, even if an exception has been occurred throughout $T_{1}$ or $T_{2}$, as the subsequent transaction object, denoted $T_{\text {next, }}$ it observes that the firstLock belonging to $T_{1}$ or $T_{2}$ has been released, but the secondLock still unexpectedly remains, thus $T_{\text {next }}$ will automatically release the lock in their secondLocks and commit the transactions.

\section{Concurrency control protocol}

Under the microservice architecture, business modules are often deployed on multiple machines in a distributed cluster to achieve scalability and high availability. When multiple microservices act as participants and concurrently execute the same group of distributed transactions, conflicts are inevitable, and they greatly affect the performance between concurrent transactions. To reduce the probability of conflict between concurrent transactions, a novel concurrency control protocol is proposed based on the $2 \mathrm{PC}$ in this subsection. Our scheme is able to avoid the additional performance overhead caused by conflicts between frequent transactions.
Similar to $2 \mathrm{PC}$, the design of the basic protocol is divided into two phases, i.e., the begin phase and the commit phase, which are summarized below.

\section{Begin phase}

When the distributed transaction process offici-ally begins, the TCM (transaction coordination manager) generates a globally unique transaction number TID for the transaction group. Then, this transaction group does not imm-ediately perform the subsequent process but instead caches it in a temporary area (such as Redis). Finally, we determine whether the transaction group matches a specific condition (which will be described in detail later); if so, the next step is performed. The key to the protocol is to maintain a graph data structure in the appropriate microservice module $M S$, which is used to record all conflict dependencies from the transact-tion group and is denoted as GraphNode. Its implementation code is shown in Table 2, in which data is the generic Java type corresponding to vertex $E$ in the Graph, and it records the key information for transaction object $T$, including the status of the transaction, which can be summarized as the three kinds of situations below.

- BEGUN. This indicates that the transaction has started execution.

- COMMITTING. This indicates that the transaction is performing the committing process.

- FINISHED. This indicates that the workflow of each transaction has been determined.

The execution order of these statuses needs to be strictly guaranteed to be serial. Thus, we restrict BEGUN to be earlier than COMMITTING and COMMITTING to be earlier than FINISHED; i.e., BEGUN < COMMITTING < FINISHED.

The field visited of GraphNode is set to the Boolean type, and it indicates whether transaction object $\mathrm{T}$ is finished with the corresponding MS. It is TRUE if done, FALSE otherwise.

The neighbourList of GraphNode corresponds to the subset of $\mathrm{V}^{*} \mathrm{~V}$ in the direct graph, denoted $E$, which is represented by the ArrayList type in Java. The sequence of all concurrent transactions in the neighbourList is recorded. For example, the $M S$ may receive a transaction request initially from transaction $T_{1}$ and then accept another request from $T_{2}$. Therefore, $T_{1}$ and $T_{2}$ are prevented from participating in the same group; they conflict and need to be added to the neighbourList.

Finally, when $T$ prepares to participate in the transaction, $M S$ does not immediately perform subsequent logical operat-ions but first enumerates all other transaction objects $T$ ' that collide in parallel with $T$ and adds them to the neighbourList. The neighbourList is 
Table 2 GraphNode data structure

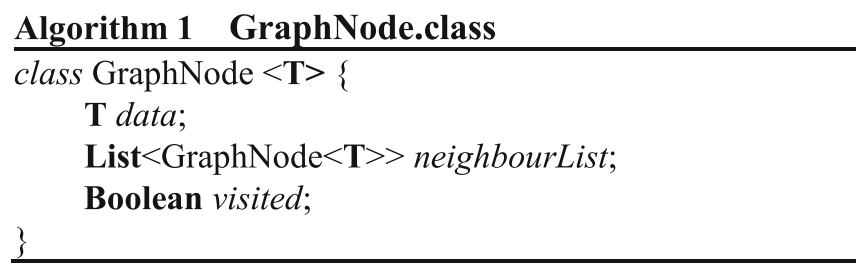

then loaded into the TCM to perform initial processing of these conflicting transactions, including aggregation and deduplication (Table 3). Finally, we save the neighbourList to the corresponding GraphNode.

\section{Commit phase}

After the begin phase, the conflicts of all transaction objects have been saved in the TCM and recorded in the corresponding GraphNode. The vertex data and the edge neighbourList belonging to the GraphNode have executed preliminary data aggregation processing. The TCM further aggregates the status of all transaction objects to preserve the latest version. As shown in the pseudo-code (Table 4), we first determine whether the status of the local $T$ is BEGUN. If so, the status can be updated to COMMITTING and synchronized to the local GraphNode.

We now describe the judgement condition mentioned in the begin phase, that is, whether the transaction object $T$ needs to be reordered. We first calculate the ancestor $T_{\text {root }}$ of $T$, and then wait for the status of $T_{\text {root }}$ to be set to COMMITTING or FINISHED. This is summarized in the following two situati-ons:

- $T_{\text {root }}$ is in the $M S$ where $T$ is located. The $M S$ will eventually receive the transaction request of $T_{\text {root }}$ through the TCM, and no additional operations are required to wait for the request.
- $T_{\text {root }}$ is not in the $M S$ where $T$ is located. At this point, the $M S$ needs to initiate an inquiry request to the $M S^{\prime}$ where $T_{\text {root }}$ is located. When the $M S^{\prime}$ observes that the status of $T$ ' is COMMITTING or FINISHED, it responds to $M S$ and returns the GraphNode to the $M S$.

The while operation is performed in both cases until all ancestors' statuses of the transaction object $T$ in the $M S$ are set to COMMITTING or FINISHED to jump out of the loop. We calculate the MS's strongly connected component (SCC) through the GraphNode. According to the definition of the $S C C$, in a directed graph GraphNode, for each pair of vertices $V_{\mathrm{i}}$ and $V_{\mathrm{j}}\left(V_{\mathrm{i}}\right.$ and $V_{\mathrm{j}}$ do not belong to the same vertex), it is guaranteed that there are paths from $V_{\mathrm{i}}$ to $V_{\mathrm{j}}$ and $V_{\mathrm{j}}$ to $V_{\mathrm{i}}$.

We utilize the classic Tarjan algorithm [22] to calculate the SCC of the GraphNode. The core design is to maintains the graph based on the depth-first search (DFS) algorithm, and each SCC is a subtree in the search-tree. The nodes belonging to the DFS that are not traversed are added to a stack. When backtracking, we determine whether the top-to-middle node is a strongly connected component. In the best case, only the vertices of the SCC belonging to the GraphNode are traversed, thus the time complexity is no more than $\mathrm{O}(\mathrm{V})$. In the worst case, all the vertices and edges in the GraphNode need to be traversed in turn, with time complexity is $\mathrm{O}(\mathrm{V}+\mathrm{E})$.

Table 3 Algorithm implementation of Begin phase

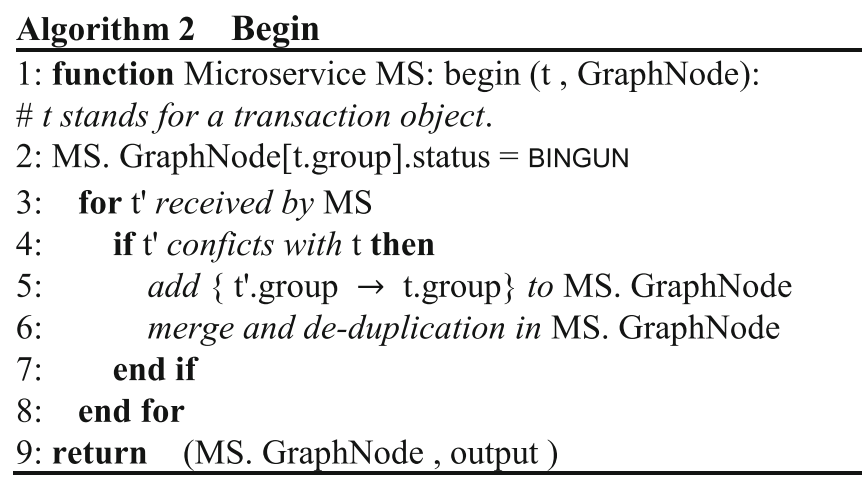




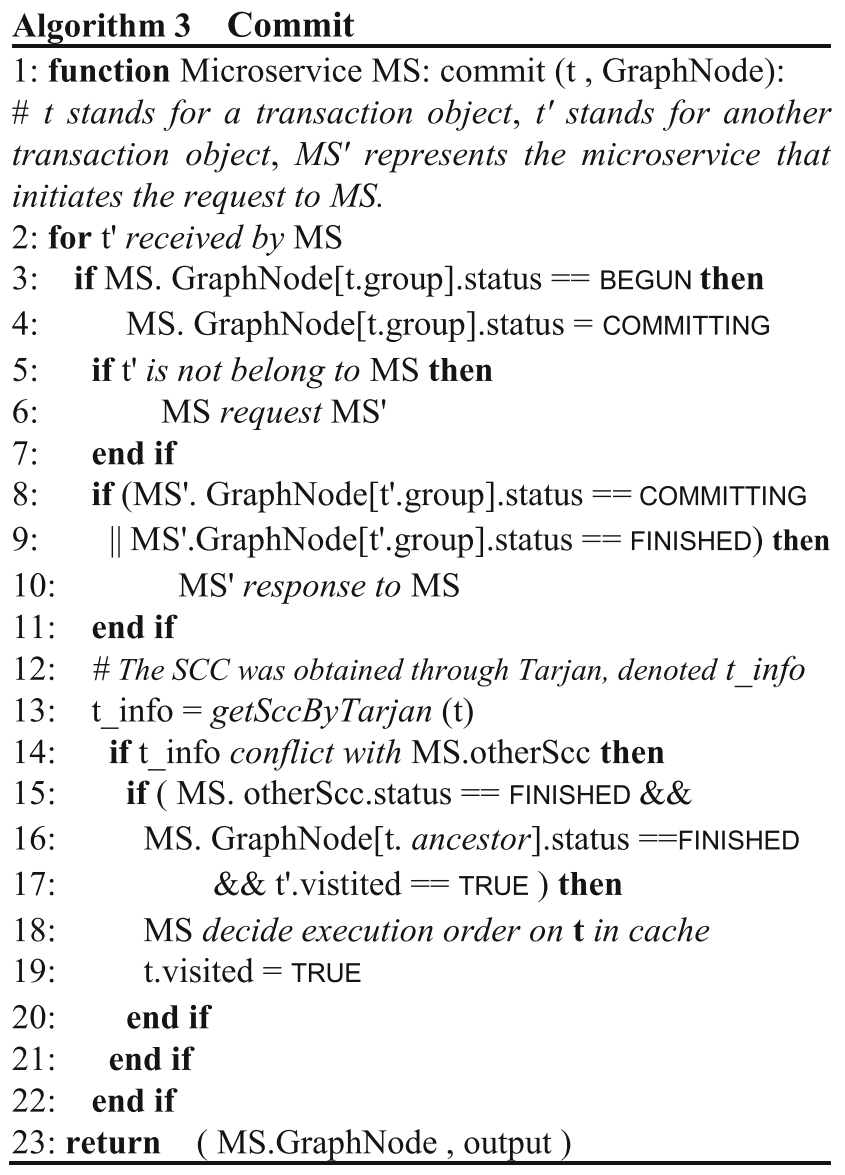

The $S C C$ of $T$ is simply referred to as $T_{\mathrm{SCC}}$. If there are no conflicts with the $M S$, the $T_{\mathrm{SCC}}$ contains only $T$ individual nodes. Otherwise, the following three conditions must be met.

- The $M S$ sets the status update in all transaction objects belonging to $T_{\mathrm{SCC}}$ to FINISHED.

- The MS waits for the status of other ancestors in GraphNode to be FINISHED.

Table 5 Algorithm implementation of SimplifyConflicts

\begin{tabular}{l} 
Algorithm 4 SimplifyConflicts \\
\hline 1: function simplifyConflicts $(\mathrm{t}$, GraphNode): \\
\# t stands for a transaction object, $t^{\prime} \rightarrow t$ is a LRCD. \\
2: for $\left\{\mathrm{t}^{\prime} \rightarrow \mathrm{t}\right\}$ to GraphNode \\
3: if GraphNode contains $\left(\left\{\mathrm{t}^{\prime} \Rightarrow \mathrm{t}\right\}\right)$.length $>$ \\
$\quad\left(\left\{\mathrm{t}^{\prime} \rightarrow \mathrm{t}\right\}\right)$.length then \\
4: remove $\left\{\mathrm{t}^{\prime} \rightarrow \mathrm{t}\right\}$ from GraphNode \\
5: end if \\
6: end for
\end{tabular}

- The $M S$ waits for the visited in its associated ancestor $T_{\text {root }}$ to be TRUE.

When all of the above conditions are met, the MS determines the eventual order based on the global transaction number TID in each $T$.

The $M S$ sets the field visited in each $T$ belonging to $T_{\mathrm{SCC}}$ to TRUE; this is executed sequentially in the order in which they are arranged. Finally, the output is sent to the TCM, and the TCM then notifies the transaction initiators of the final execution result. We can determine the time complexity of algorithm 3 as $\mathrm{O}(\mathrm{n} *(\mathrm{~V}+\mathrm{E}))$ in the worst case and $\mathrm{O}\left(\mathrm{n}^{*} \mathrm{~V}\right)$ in the best case (where $\mathrm{n}$ stands for the number of $M S s$ ).

\section{Correctness}

In this subsection, we present the correctness verification of $2 \mathrm{PC} *$. We utilize the formal specification language TLA+ [23] to validate the transaction's locks through the protocol, ensuring that $2 \mathrm{PC}^{*}$ is strictly serialized. We only show the core TLA+ of the protocol, a 
more rigorous version of the TLA+ language is available at [24].

\section{Constant}

We set the two invariants in the transaction to constants, i.e., the data of all transaction participants, denoted as value, and the participant transaction object, denoted as $R M$.

\section{Variable}

We represent all transaction status in $R M$ as the variable rm_status. The current version sequence number is represented by the variable rm_v, which includes a collection of all the states of the transaction, i.e., \{"beginning", "preparing", "precommit", "committed", "cancel"\}. We define the global version sequence ascend_v, which is a snapshot and is always increasing. The information about two locks in $R M$ that control the version, firstLock and secondLock, is represented by the variable rm_lock.

\section{Begin}

At this point, the transaction status rm_status is at "beginning" and its next state is expected to be "preparing". The data rm_value in the transaction object is obtained by the getVal method.

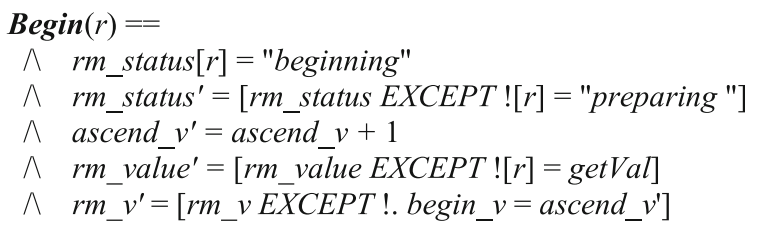

\section{Loading}

At this stage, rm_status is in the "preparing" state and its next state is bound to be "precommit". We determine whether the condition of resetting lock is met. If so, we then perform the resetLock process. Otherwise, the next state of rm_status is set to "cancel".

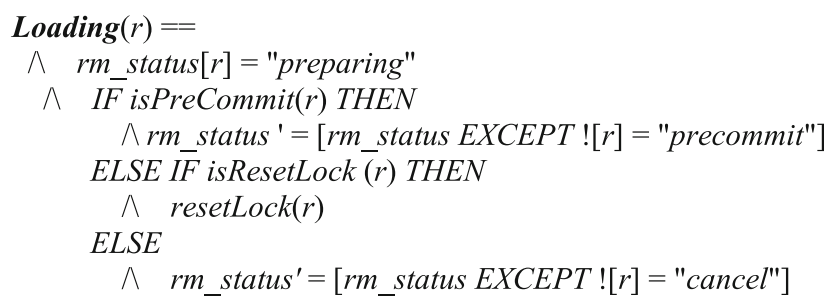

\section{PreCommit}

We present the TLA+ language for the pre-commit phase of the transaction. At this point, rm_status is "precommit", and we determine whether the second-commit of the transaction is performed, if so, ascend_v needs to be incremented, and next state of $r m_{-} v$ is restricted to be the latest value of ascend_ $v$, and rm_status's next state is limited to "committing". Otherwise, we need determine whether these transaction's values are all locked, if so, we perform the allLock method to lock all values. Otherwise, we set rm_status to "cancel".

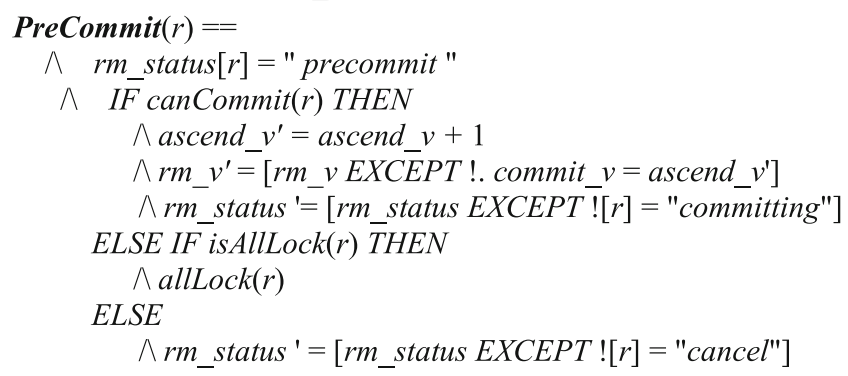

\section{SecondCommit}

Finally, we present the TLA+ language for the secondcommit of the transaction. We determine whether the first value of the transaction can be committed, i.e., isCommitFirstVal, if so, we can commit the transaction and then set rm_status to "committed". Otherwise, the transact-tion aborts and we set rm_status to "cancel".



Next

The entire validation process follows the four steps above.



\section{Consistent}

Finally, we present the consistency constraint for the transaction. These two constraints are met: the version sequence number committed_v for all committed transactions satisfies the ascending ordering rule, and the lock of first_ $v$ in $R M$ can be released when the transaction committed succe-ssfully.

We run the complete TLA+ language in the TLC [25] tool and analyze the result as shown in Fig. 1. It generates 1296 states, of which 324 is distinct. More importantly, based on the results, i.e., "No error has been found", which can prove that the $2 \mathrm{PC} *$ protocol will not occur deadlock and ensure the strict serialization during the transaction process.

\section{Simplifing NeighborList of GraphNode}

As the number of transaction participant increases, the amo-unt of data stored in the GraphNode becomes increasingly cumbersome, which significantly affects the subsequent wor-kflow and potentially makes it unavailable. For example, the Trajan algorithm is used to calculate the SCC steps. However, much useless information 


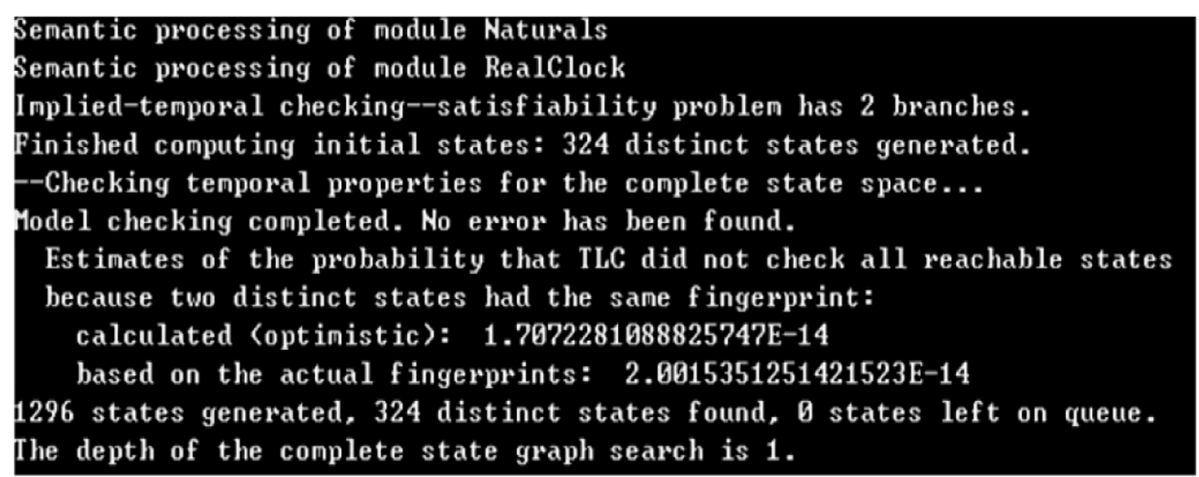

Fig. 1 TLA+ result with running in TLC

has been recorded in the Graph-Node, mainly related to the concurrent conflict dependencies of transaction objects, i.e., the neighbourList, which is irrel-evant for the subsequent process. Moreover, it is unnecessary to transfer the entire GraphNode between micro-services. To solve these problems, we optimize the runtime protocol further.

\section{LRCD design}

To simplify the neighbourList belonging to the GraphNode, only the least recent conflict dependence (LRCD) between transactions needs to be recorded Table 5 . The $L R C D$ is defined as follows: for any conflicting relationship path $T^{\prime} \rightarrow T$ in GraphNode, if the path $T^{\prime} \Rightarrow T$ does not exist in GraphNode, the number of paths $T^{\prime} \Rightarrow T$ is not less than two, and $T^{\prime} \rightarrow T$ is called an $L R C D$ in GraphNode. According to the explanation in Fig. 2 below, $T 1 \rightarrow T 2, T 2 \rightarrow T 3$ is an $L R C D$, while $T 1 \Rightarrow T 3$ is not an LRCD; thus, $T 1 \Rightarrow T 3$ can be removed. The location of Table 5 Algorithm implementation of SimplifyConflicts

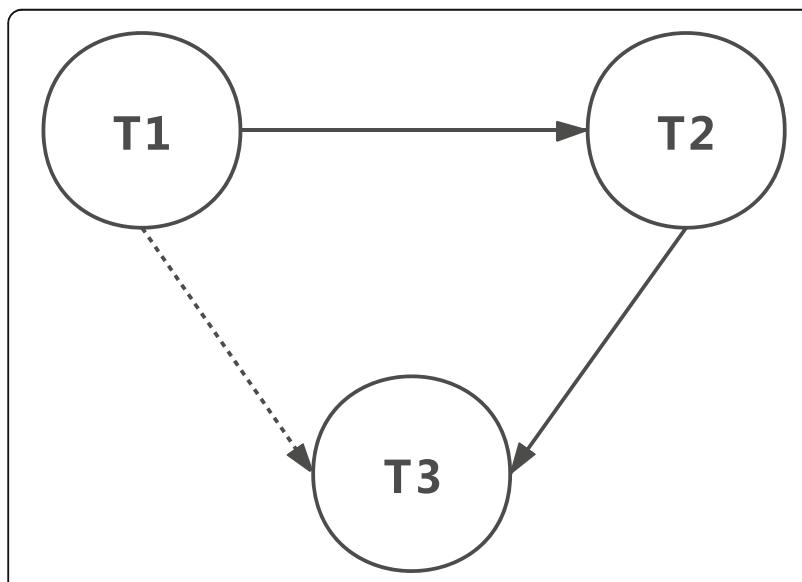

Fig. 2 A LRCD demo
In the original protocol, the following three locations need to be simplified in the neighbourList and saved only as $L R C D$ s:

In the original protocol, the following three locations need to be simplified in the neighbourList and saved only as $L R C D s$ :

- In the begin phase, the TCM received a response from the MS and then simplified the neighbourList that belonged to the response message.

- During the commit phase, the $M S$ received a request from the TSM. The MS simplified the neighbourList in the request body with its local value.

- During the commit phase, the MS received an inquiry response from the other $M S^{\prime}$. The $M S^{\prime}$ simplified the neighbourList in the request body with its local value.

\section{Simplify MS swaps with GraphNode steps}

For the $M S$ and $T C M$ corresponding to $T$, we only need to obtain the GraphNode of T'. Therefore, the MS and $T C M$ only need to obtain the SCC whose status is FINISHED and whose GraphNode contains all transaction objects.

Similarly, when the $M S$ receives the query request from $M S$, the $M S$ first detects the status of the GraphNode under the local environment. If the status is not FINISHED, we calcu-late the $S C C$ for all the transactions contained in GraphNode belonging to the MS. Otherwise, if status becomes FINISHED, this means that $T$ and its $S C C$ have been obtained by the $M S$ and its response is returned directly to $M S$ '. The process is shown in pseudo-code in Table 6 below.

\section{Fault tolerance}

To achieve fault tolerance, we persisted the transaction logs in each coordination and transaction participant service to the disk. Moreover, we used the Paxos-based 
Table 6 Algorithm implementation of SimplifySwapGraphNode

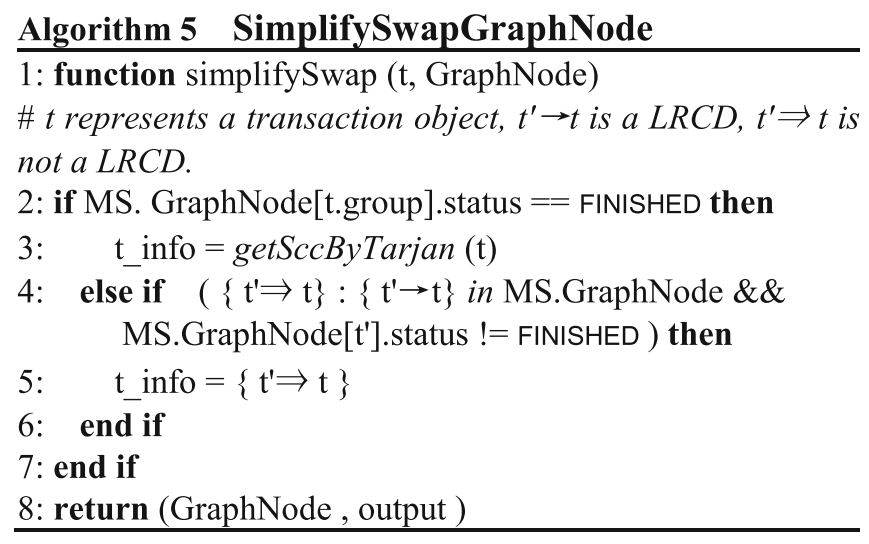

replication protocol [26] to synchronize the log data across multiple machines.

First, we execute a scheduled thread-pool task, denoted as STPT. STPT polls the logs in the disk at intervals. It filters out the key information in the failed transactions, including the TID that identifies the transaction, and the list of participating transactions. Then, we push the information into a circle message queue $(C M Q)$. Finally, the $C M Q$ repeats the request to the corresponding business method through the asynchronous polling until the response returns successfully.

The CMQ design is shown above (Fig. 3), it is a circular message queue with a high latency and has 2000 slots. Each transaction compensation can be regarded as a task that is added to the Set without repetition, i.e., Set<Task $>$. We then push the Set $<$ Task $>$ into the tail of the $C M Q$. There are two key pieces of information stored in Set, i.e., layer_num and Function < Task>. The layer_num represents the number of CMQ layers in which the task resides, and the Function $<$ Task $>$ indicates the target function of the task. The CMQ uses a pointer to specify the index of the currently running task, denoted cur_index.

\section{Reliability and idempotency}

We use a local message table to record the relevant data of the transaction compensation task, which includes the
TID for the transaction, and the current state of execution (i.e., status). To ensure reliability, The TCM returns the result of the asynchronous message with the field status record and sets it to TRUE if successful and FALSE if not. Then, the failed transaction steps need to be pushed to the next task queue until it is compensated for successfully. To ensure idempotency, the TCM determines whether the message is duplicated by the TID. If the TID already exists and its status is TRUE, this step is skipped. Moreover, the TCM provides a remote message record query interface, thus the transaction participant can invoke this interface to determine whether the current message has been consumed. If so, this compensation process is skipped.

\section{Implementation}

We implemented a middleware solution with transactional in distributed microservices support using 2PC*. We design the annotation @TxTransactional to inject distributed transactio-nal functionality into specified microservices business with spring's AOP (aspect oriented programming), which achiev-es decoupling from the original business module. Obviously, our prototype consists of three functional modules, i.e., transaction initiator, transaction actor, and transaction coordinator. In particular, their network communication is implemented through high-performance Netty framework.

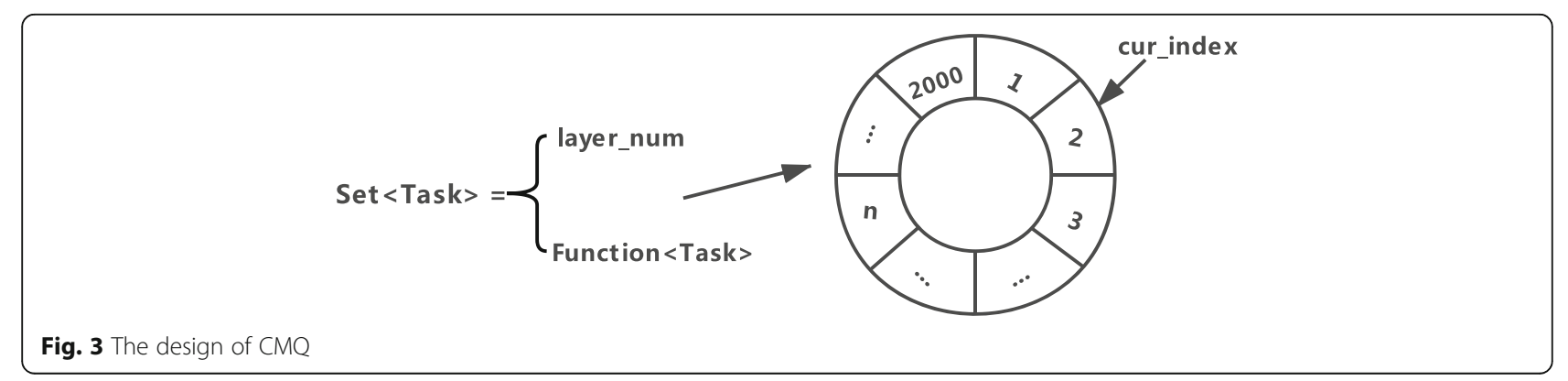




\section{Transaction initiator}

The role of the transaction initiator is an active part of the process. In addition to creating transaction group information and executing the local transaction, it also notifies the coordi-nator to perform commit or rollback operations on the trans-action group (Fig. 4). The implementation process can be summarized as follows.

1) Generating the transaction group identifier TX_ID through the snowflake algorithm to ensure that it is unique in the entire distributed system, and then creating a new transaction group. If this step is successful, we can execute step 2. Otherwise, we just throw the runtime-exception and end the process.

2) We determine the transaction propagation mechanism type of the initiator. If it is PROPAGATION_NEVER, which means that there is no transaction requirement from the initiator. If it succeeds, we perform step 3. Otherwise, end the process. If the type of propagation is PROPAGATION_REQUIRES_NEW, it means that the new transaction was initiated. We similarly execute the transaction group's pre-commit process, and if successful, we perform step 4, otherwise, jump to step 5.

3) Following step 2 above, there is no transaction request in this initiator, and after performing the pre-commit, the coordinator is asynchronously notified to complete the second-commit. We adopt the CompletableFuture interface [27] provided by the JDK1.8. When these multiple threads attempt to complete or cancel it at the same time, only one thread is guaranteed to succeed. In practice, we use the runAsync, a method without a return value, to construct the initiator and coordinator as Netty transmission object in the asynchronous mode, then complete the second-commit's notification step between the initiator and coordinator.

4) Similar to step 3, we also use the CompletableFuture to asynchronously notify the coordinator to execute the second-commit after the transaction group pre-commit succeeds. Because the transactional requirement of the initiator is newly opened, it must first commit the local transaction, thus we can adopt the

PlatformTransactionManager (PTM) interface [28] provided by Spring to execute it.

5) In this situation, the transaction group failed during the pre-commit phase and the initiator's transaction is newly opened. Then, we perform the local transaction rollback, executing the PTM's rollback function and closing the process.

6) If some unexpected exceptions occurred in steps 4 or 5, as shown in the dotted box of Fig. 4. First, we use the PTM's rollback function to complete the local transaction rollback. The coordinator is then asynchronously notified by CompletableFuture to complete the rollback of the transaction group.

\section{Transaction actor}

The transaction actor is a passive role in distributed transacti-on processing. Its main functions include adding the micro-service's business module to the distributed

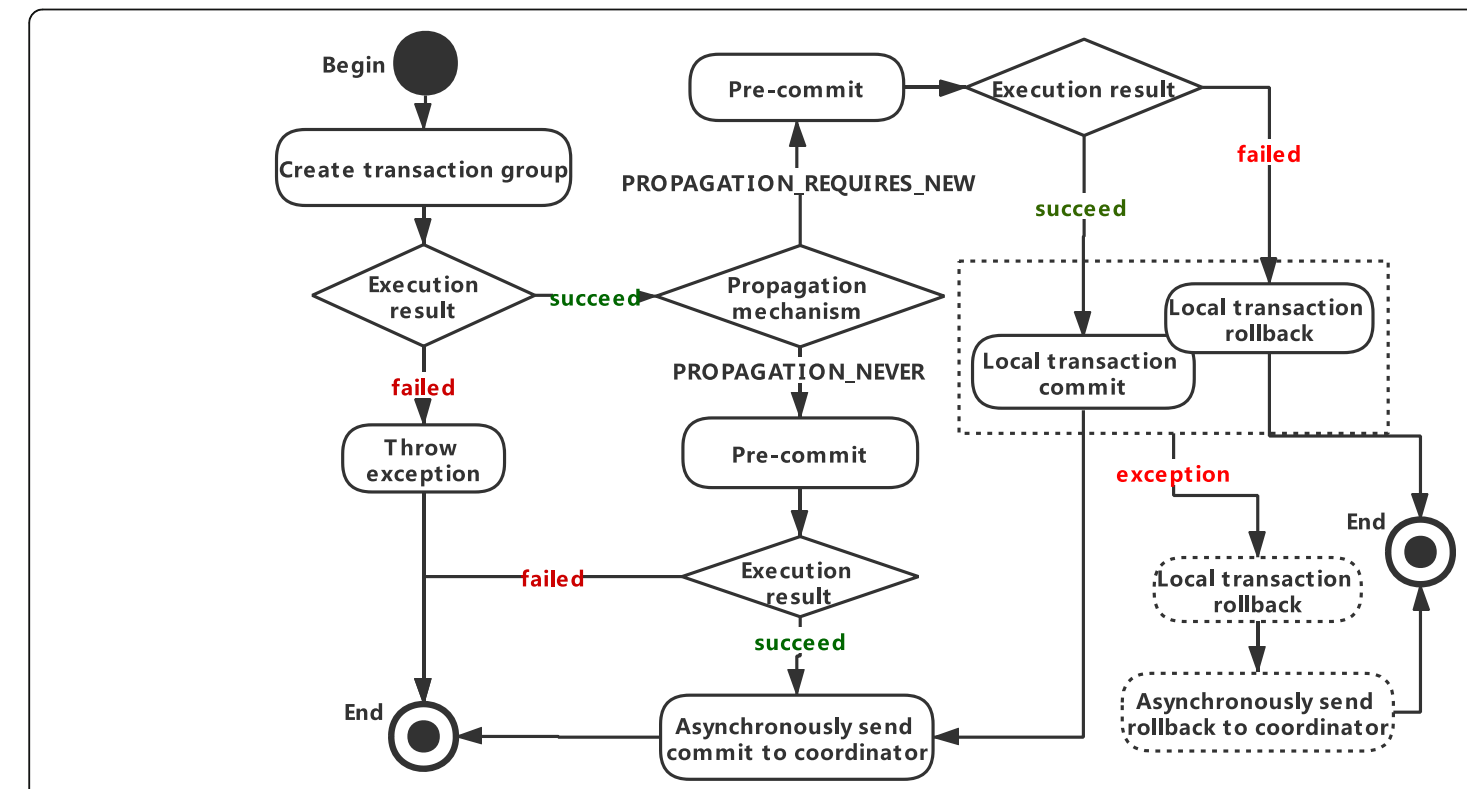

Fig. 4 The process of transaction initiator 


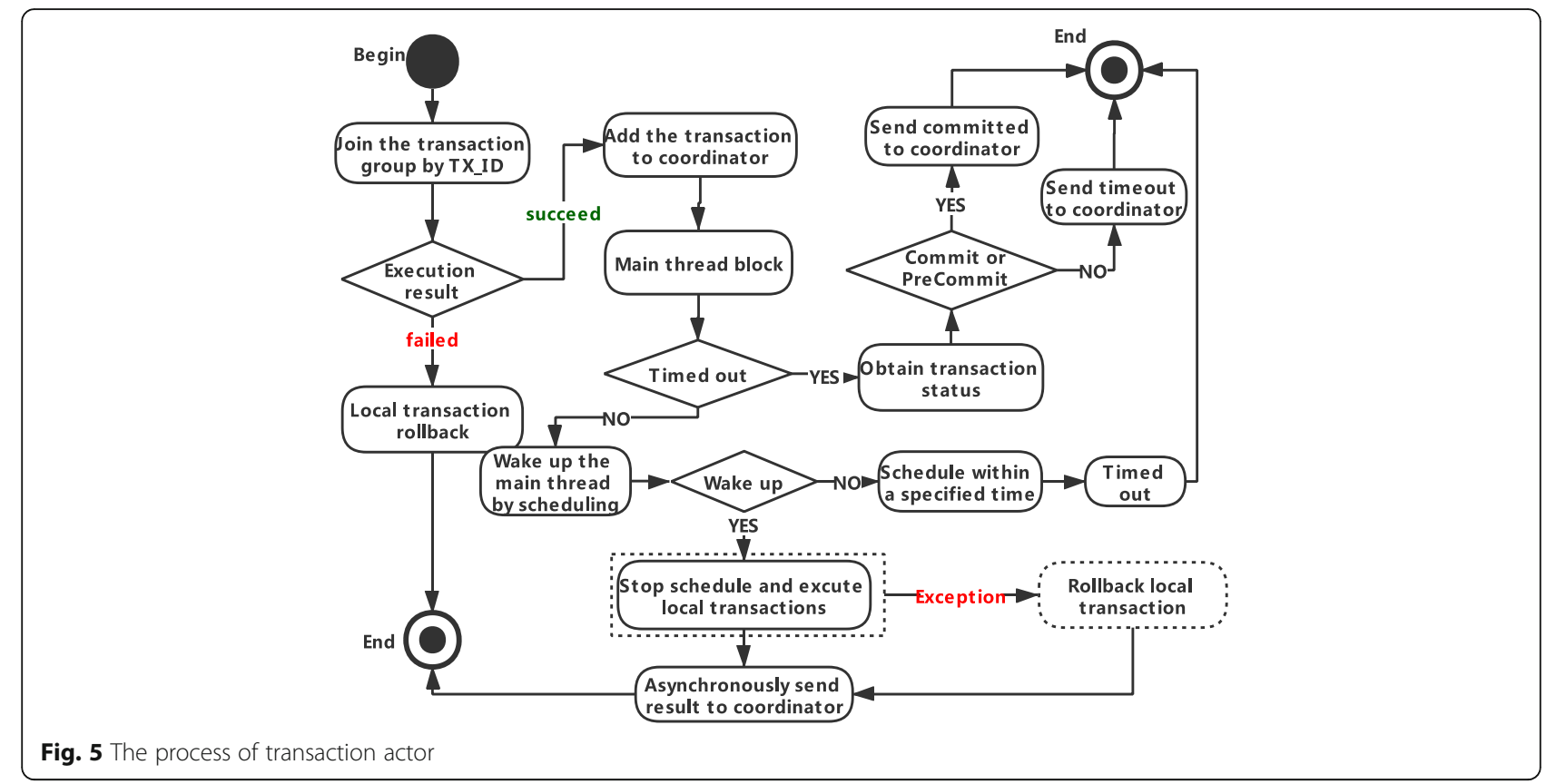

transaction group and completing local transactions through the coordinator's instruction (Fig. 5). The process of the transaction actor can be summarized as follows.

1) The actor joins the corresponding transaction group according to TX_ID. If the execution fails, the local transaction needs to rollback and then the process should be terminated. Otherwise, it initiates an addto-transaction request to the coordinator. It's similar to the initiator, the communication between actors and coordinators is also based on Netty.

2) At this point, the initiator's thread is blocked. We use the interface provided in JDK called ReentrantLock [29] to lock the main thread and wake it up in combination with Condition's signal method. The initiator then waits for the coordinator's response within the specified time threshold, denoted WAIT_TIME_OUT. If the response time is no more than WAIT_TIME_OUT, step 3 is executed directly. Otherwise, we need to perform step 6.

3) We then create a scheduled task to wake up the main thread at specified intervals within the time threshold denoted TASK_TIME_OUT. If this process is successful, the task can be closed and step 4 is executed. Otherwise, we need to skip to step 5 .

4) Following step 3, the subsequent process is based on the coordinator's response, which is obtained from the thread's asynchronous callback function. If the response is second-commit, we first commit the local transaction, and then the output is asynchronously notified to the coordinator via Netty. Otherwise, the local transaction needs to rollback, and then also asynchronously notify the coordinator of the result through Netty.

5) Continuing with step 3 , we repeat the scheduling task for the specified time TASK_TIME_OUT until it succeeds. Otherwise, a timeout occurs and the process can be terminated.

6) Next, following step 2, the coordinator's response is timed out. The initiator needs to proactively obtain the transaction group's status through Netty. If the status is either pre-commit or second-commit, the transaction group has successfully committed and then the actor sends the commit notification asynchronously to the coordinator. Otherwise, there are some exceptions have occurred in the execution, and actor can asynchronously send the timeout exception notification to the coordinator. Finally, we need to wake up the main thread that is blocked by the Condition's signal.

7) If some exceptions occurred to the initiator during the process of the local transaction's commit, as shown in the dotted box in Fig. 5, we should rollback this transaction and notify the coordinator asynchronously through Netty.

\section{Transaction coordinator}

The transaction coordinator is at the core of the hub in the distributed transaction processing. On the one hand, it deals with the corresponding business according to the notification requested by the initiator and the actor. On the other hand, it sends the instruction response to the 
initiator and the actor at a specified time. We first design the transaction management interface TransactionManangerService, denoted TMS. TMS provides basic functionality related to the persistence of the transaction, i.e., CRUD (Create, Retrieve, Update and Delete). We then describe the transaction coordinator's two core functions, i.e., precommit and rollback.

\section{PreCommit}

The process can be divided into the following steps.

1) With the updateItemStatus method of TMS, we update the current transaction's status to COMMIT.

2) Through the listByGroupId method in TMS, we obtain the list of all transaction objects under the current transaction group number TX_ID, denoted items. Determine whether the items are empty. If so, it can terminate the process. Otherwise, we execute the next step.

3) Following the above steps, we now perform specific preliminary filtering of items. The filtering principle is that we remove the transaction objects that have been committed by the initiator from the items, that is, avoiding duplicate the communication between these transactions. By the filter function, we divide items into a list of transactions under the local domain environment, denoted currentItems, and another list under other domains, denoted elseItems.

4) Detecting whether Netty's channels of currentItems are activated. If so, we run the excuteCommit method to commit the transaction, otherwise, run the specific excuteRollBack method to complete the transaction rollback. The excuteCommit and excuteRollBack will be described later.

\section{Rollback}

Similarly, its process can be divided into the following steps.

1) We update the current transaction's status to ROLLBACK through the updateItemStatus method in $T M S$

2) It is the same as described in step 2 of the PreCommit.

3) Similarly, it is the same as described in PreCommit's step 3.

4) Finally, we execute the excuteRollBack method to complete the transaction rollback.

In the process of PreCommit and Rollback, they both need to run two specified methods, i.e., excuteCommit and excute-Rollback. Next, we describe the implementation details for each.

\section{ExcuteCommit}

This method applies to the distributed tran-saction's commit process that is divided into the following steps.

1) First, we iterate through the list of local transaction groups to be committed, i.e., currentItems.

2) Then, we build Netty's ChannelBean object and load it in the HeartBeat, and set the transaction status to COMMIT.

3) Determining if the channel in the ChannelBean object is empty. If so, we record the transaction object's TX_ID in the Error log. Otherwise, pushing the HeartBeat to Queue and refresh it.

4) Finally, we execute the remote request method with the elseltems and set the transaction status to COMMIT. Because these coordinators are clustered, thus the channels in elseItems' transaction objects may be connected to different coordinators. There are two functions in the remote request method. On the one hand, we observe the status of the transaction coordinator channel under the local domain and notify it to perform the transaction commit. On the other hand, we connect to the clusters of transaction coordinators under other remote domains and similarly notify them of committing transactions.

\section{ExcuteRollback}

It is responsible for the rollback process of distributed transactions, which can be roughly divided into the following steps.

1) First, we determine whether the currentItems are empty. If it matches, we just skip to the last step. We then load the list of transaction groups into the specified ThreadPool array, i.e., CompletableFuture, which asynchronously performs the tasks of the subsequent multi-transaction groups. To achieve this, we then execute the asynchronous method in the CompletableFuture, i.e., runAsync. Meanwhile, we build the Netty's ChannelBean object, load it into a HeartBeat, and set status to ROLLBACK.

2) Determining if the channel exists in the ChannelBean. If not, we just skip this step, otherwise, execute the writeAndFlush method for ChannelBean's channel. Finally, we can push the HeartBeat object to Queue and refresh it.

3) Until all transaction objects have been loaded into Netty's channel and have been executed asynchronously through CompletableFuture. At this point, we execute the allof method, which can acquire the execution result of all transaction objects. 
4) Finally, the approach is roughly similar to the last step in ExcuteCommit. The only difference is that we set the transaction status to ROLLBACK and perform the transaction rollback.

\section{Evaluation}

\section{Experimental setup}

In order to minimize the extra impact of CPU's performance bottlenecks on the experiment, we chose higher performance machines to build service clusters. Each machine has an eight-core $2.7 \mathrm{GHz}$ Intel Core i7 with 8 GB RAM and 500GB SSD. Therefore, we have achieved much higher throughput when running on a local testbed with faster CPUs.

\section{Experimental case}

We applied our middleware solution based on $2 \mathrm{PC}^{*}$ to the microservice case, that is, the online e-commerce transaction platform MSECP of an Internet company. In this paper's experimental case, we primarily consider three microservices, i.e., the OrderMicroservice (COMS), the StockMicroservice (CSMS), and the AccountMicroservice $(A M S)$. The distribu-ted transaction process between the microservices can be summarized as follows (Fig. 6): A user initiates an order creation request from COMS, which then invokes CSMS and AMS through an RPC to complete the business of item out-bound and account deduction, respectively. Only when the business of these three microservice modules is successfully executed can we return a successful response to the user and commit transactions in the respective microservice modules. Otherwise, if exceptions occurred in one of these micro-services, the user is notified that the purchase failed; thus, the transactions of the respective microservices need to be rolled back immediately.

We adapted the middleware solution to two popular micro-services frameworks, i.e., Spring Cloud and
Dubbo. In this section, we only present the experimental case of Dubbo, whose overall architecture is shown in Fig. 7. We adopted Dubbo to implement the three microservice modules, i.e., COMS, CSMS and AMS, which deployed three physical mac-hine clusters for each module. They completed the service registry on the Zookeeper [30] and used the Nginx [31] server to complete the service's reverse proxy. To ensure the high availability of the coordination service (denoted as CS), we adopted Eureka [32] to achieve service registry and service renewal and transferred the transaction entities to the cluster-ed Redis database. In particular, the network communication between the $C S$ and multimicroservice modules is based on Netty's persistent connection.

\section{Functional experiment}

In this subsection, we design a specific test case with high randomness and a wide range to prove the functional reliab-ility of our middleware solution with distributed transactions in the multi-microservice modules, which can be described as follows.

- In COMS, the unit-price of item is randomly generated between $\$ 100$ and $\$ 10,000$, and the order number is set to be randomly generated between 1 and 1000 , and the quantity purchased by the user is randomly generated between 1 and 100 . We initialize the account balance in $A M S$ to be $\$ 0$, and the amount of stocks in CSMS to be 0.

- In COMS, CSMS, and AMS, we embed a runtime exception to abort the transaction process in the business code for order numbers 100, 200 and 500, respectively.

- Then, we continuously invoked the createOrder API interface, making a total of approximately 50,000 calls.

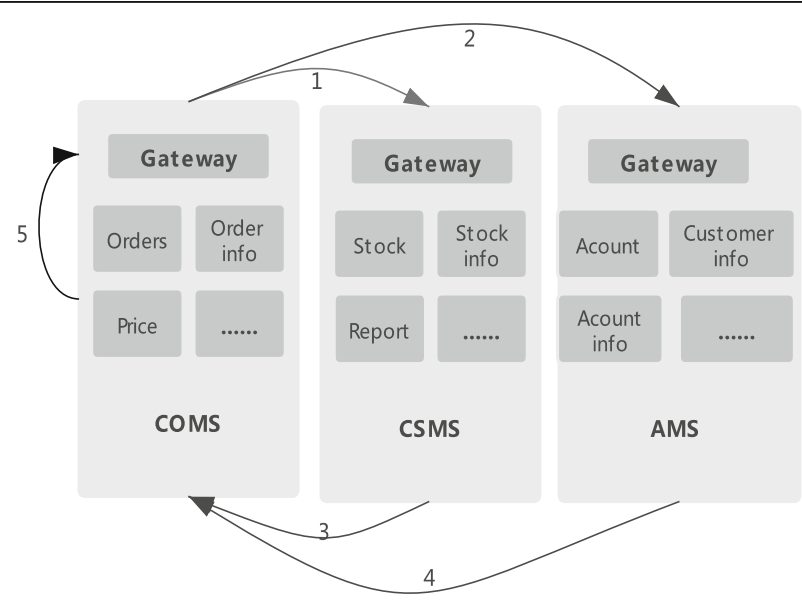

1. COMS starts the order creation business, and RPC calls CSMS to

complete the corresponding stock business

2. COMS RPC calls AMS to

complete the payment business

3. CSMS returns the execution result of stock deduction (success/failure)

4.AMS returns the deduction result for the account (success/failure)

5.Display the final result to user, generate order information

Fig. 6 A case of Ctrip MSECP 


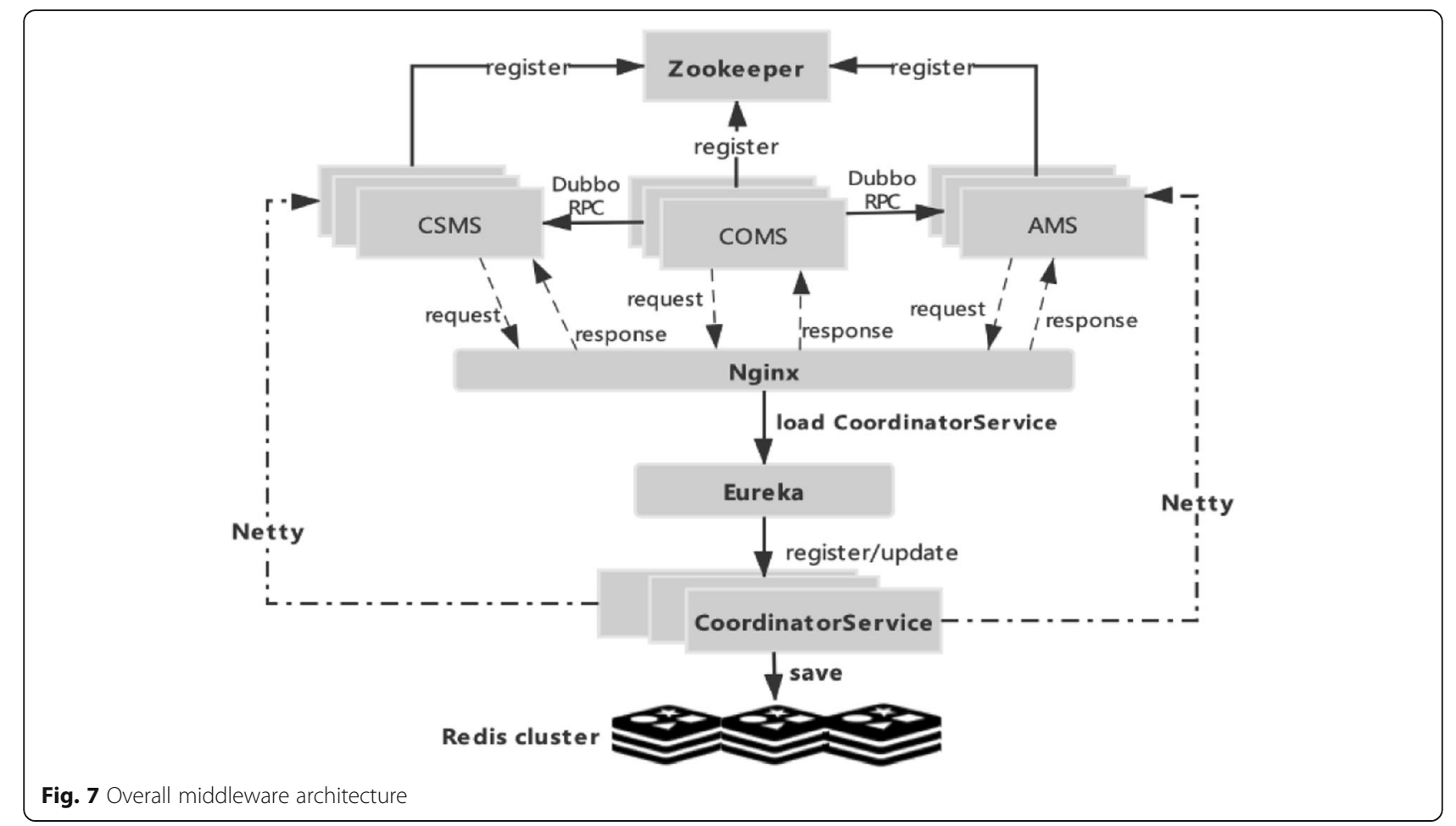

- Finally, we determined that the data-consistent distributed transaction in this case should meet the following two conditions: the sum of the order amount and the account balance should be 0 , and the sum of the order quantity and stock quantity should be 0 .

According to the five groups of experimental results shown in Table 7 above, the createOrder interface was called approximately 5000 times, and its orders were successfully created approximately $2,450,000$ to 3,180 , 000 times. More importantly, all experimental data met the above two condi-tions, i.e., Order amount + Account balance $=0$; Order number + Stock number $=0$. Therefore, our scheme is able to achieve consistent distributed transactions for multiple applications involving microservices.

\section{Performance experiment}

In this section, the evaluation of our scheme explores three key questions:
1) How does the throughput and latency of the optimized 2PC* compare with the traditional 2PC approach at varying levels of contention across microservices?

2) Can $2 \mathrm{PC}$ " guarantee its commit rate under the scenario of high-level contention?

3) Can our optimization scheme compensate for failed transaction steps?

\section{Throughput}

In this experimental case, we evaluated the throughput performance of our scheme through the indicators of TPS (transactions per second). We compare the TPS of the optimized protocol $2 \mathrm{PC}^{*}$ and $2 \mathrm{PC}$ and adopt the number of local transactions of the database (i.e., Mysql) as a reference. We ran 10, 20, 50, 100, 200, 300, and 500 concurrent threads to call COMS's CreateOrder interface, and each thread exe-cuted 10 comparison experiments. Finally, we calculated and recorded the TPS averages.

Table 7 Data consistency experiment results

\begin{tabular}{llllll}
\hline No. & Call times & Order amount & Account balance & Order number & Stock number \\
\hline $\mathbf{1}$ & 50,142 & $24,842,305,224$ & $-24,842,305,224$ & $2,850,098$ & $-2,850,098$ \\
$\mathbf{2}$ & 51,203 & $27,514,201,548$ & $-27,514,201,548$ & $3,183,214$ & $-3,183,214$ \\
$\mathbf{3}$ & 50,893 & $22,870,237,842$ & $-22,870,237,842$ & $2,581,249$ & $-2,581,249$ \\
$\mathbf{4}$ & 50,071 & $24,847,024,109$ & $-24,847,024,109$ & $2,708,291$ & $-2,708,291$ \\
$\mathbf{5}$ & 50,019 & $23,787,312,291$ & $-23,787,312,291$ & $2,457,219$ & $-2,457,219$ \\
\hline
\end{tabular}




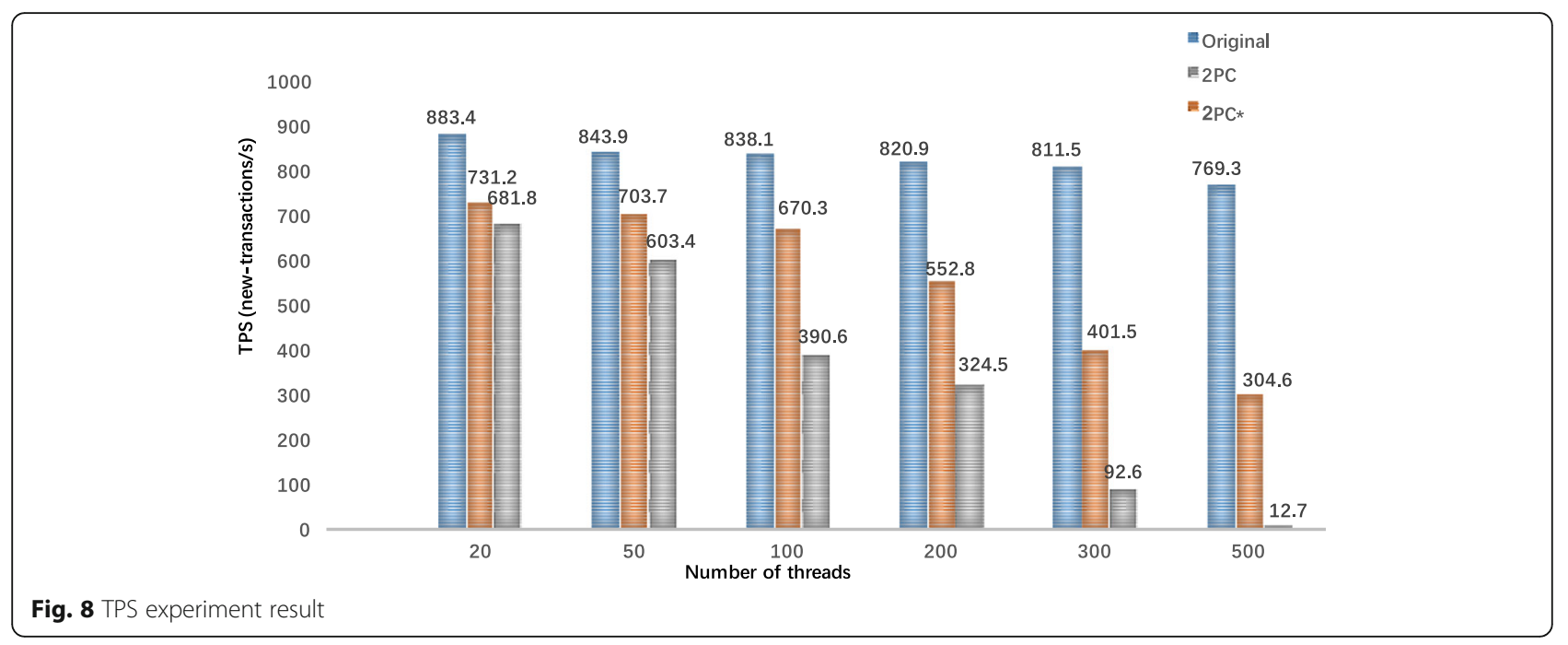

Through the experimental data shown below (Fig. 8), when the number of concurrent threads is between 20 and 50, the distributed transactions are under low-level contention. Compared with $2 \mathrm{PC}$, the $2 \mathrm{PC}$ protocol has no significant advantage in terms of the TPS metric. However, when the number of concurrent requests increases from 50 to 100, the contention of the distributed transactions increases from low to moderate, and the performance gap between $2 \mathrm{PC}^{*}$ and $2 \mathrm{PC}$ gradually widens. At this point, when the number of concurrent threads is between 100 and 200, the TPS of $2 \mathrm{PC}^{*}$ can still be maintained at a relatively optimistic level, i.e., 670.3 and 552.8 new transactions/s, respectively, while 2PC's TPS is reduced to 391.6 and 324.5 , respectively. Compared to transactions from the local database, 2PC*s TPS drops by $\sim 32.7 \%$ when the number of concurrent requests is 200 , while $2 \mathrm{PC}$ 's drops by $\sim 60.5 \%$; the throughput of $2 \mathrm{PC} *$ improved by $\sim 70.4 \%$ compared to the original approach under moderate contention.

In the scenario with high-level contention, i.e., when the number of concurrent requests is from 300 to 500 , $2 \mathrm{PC}^{*}$ shows significant advantages. Under the scenarios with 300 concurrent requests, 2 PC*'s TPS reduces to 401.5 new trans-actions/s, which is still half the performance of the local transactions, while 2PC's TPS reduces to only 92.6. In parti-cular, when the number of concurrent requests peaked at 500, the throughput of $2 \mathrm{PC}$ reached a performance bottleneck, while our scheme could still scale out. As the experimental data show, the transaction throughput performance of $2 \mathrm{PC}^{*}$ is still quite high compared to that of 2PC; the TPS values are 304.6 new transactions/s and 12.7 new transactions/s, respectively. We abandoned the low-performance synchronous blocking lock in the control of transaction resources and repl-aced it with a novel second-level asynchronous lock, i.e., the SAOL, which can greatly reduce the blocking caused by the surging number of transactions; this is the key factor in the obvious advantage of the TPS performance of $2 \mathrm{PC}^{*}$.

In summary, our scheme has a significant improvement of throughput compared to the traditional approach, especially in scenarios of high-level contention. In other words, when the number of concurrent requests from users reaches a peak, the throughput of $2 \mathrm{PC}^{*}$ can still maintain excellent perfor-mance, and it can be applied to highly concurrent requests for microservices with distributed transactions.

\section{Latency}

Transaction latency is another key indicator in our evaluation. We adopt the service's RT (response time) parameter to evaluate the latency performance of $2 \mathrm{PC}$. Similar to the experimental case in the "Throughput" sub-section, we ran $10,20,50,100,200,300$, and 500 concurrent threads to call COMS's CreateOrder interface, performed 10 comparison experiments for each thread and calculated their RT averages.

Through the analysis of the experimental results (Fig. 9), when the transactions are under low-level contention, i.e., the number of concurrent requests is between 20 and 50, 2PC*s RT has no obvious advantage compared with that of $2 \mathrm{PC}$; for example, when the number of requests reaches 50 , our scheme only reaches $18.3 \%$ improvement. The $\mathrm{RT}$ of $2 \mathrm{PC}$ is more sensitive to the increase in contention. When the number of concurrent requests grew to 200, the transactions reached moderate-level contention, and the latency of $2 \mathrm{PC} *$ achieved a significant performance advantage. In detail, when the number of concurrent requests reaches 100 , the $\mathrm{RT}$ of $2 \mathrm{PC}^{*}$ is only half that of $2 \mathrm{PC}$. Compared to the original businesses' RT value, our scheme drops by $\sim 39.6 \%$. The latency superiority of $2 \mathrm{PC}^{*}$ under high 


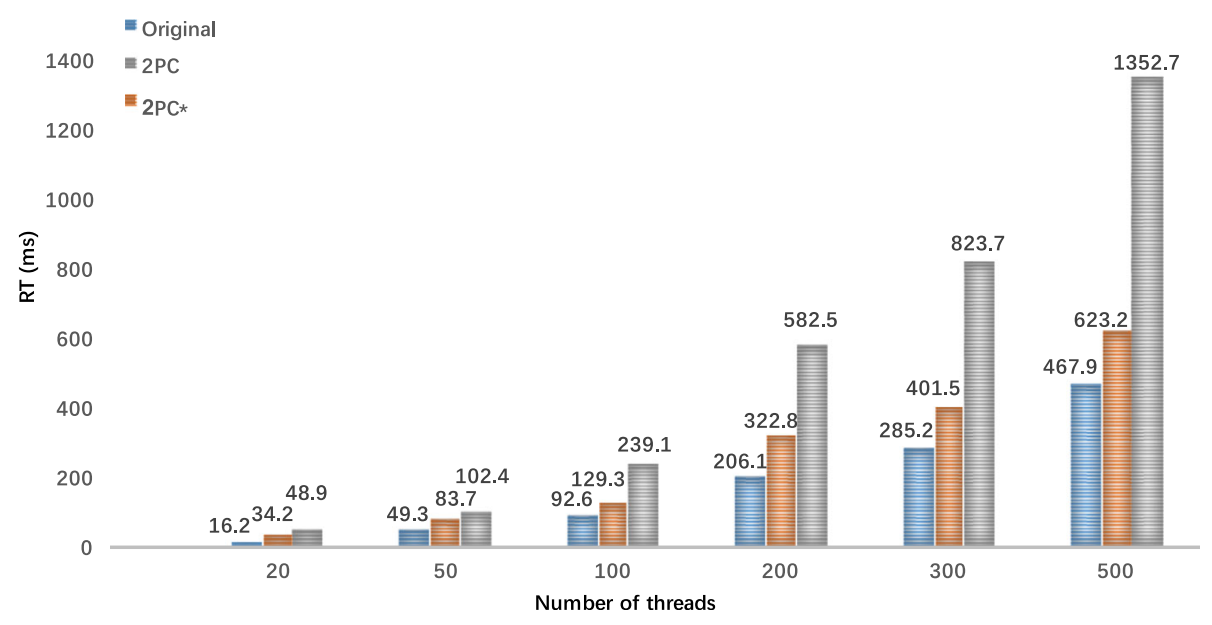

Fig. 9 RT experiment result

contention becomes more obvious. When the number of concurrent requests reaches 300 , the $\mathrm{RT}$ of $2 \mathrm{PC}$ is 823.7 $\mathrm{ms}$, which is 2.89 times that for the original business, while our scheme only increases to $401.5 \mathrm{~ms}$. In particular, when the contention reaches its peak, $2 \mathrm{PC}$ is no longer suitable for the distributed transaction across microservices because its RT surpasses one second; it is $1352.7 \mathrm{~ms}$. 2PC* is less sensitive to the increase in contention; the RT drops by $\sim 33.4 \%$ with the original business and the latency is reduced by more than half compared with that of $2 \mathrm{PC}$.

Similar to TPS, the factors affecting RT performance are closely related to the blocking rates between transactions. In the runtime protocol, $2 \mathrm{PC} *$ adopts an optimization algorithm based on a directed graph to aggregate and reorder the depen-dencies between transactions, which is able to reduce the conflict probability and avoid deadlocks and aborts between transactions. Additionally, the specific implementation utiliz-es the persistent connection network communication mode of the Netty framework, and we choose asynchronous threads in the coding; these are the key factors that show the obvious advantages of our proposed scheme in the experimental results of latency.

In summary, $2 \mathrm{PC}^{*}$ demonstrates a better latency performa-nce with high-level contention than the traditional approach. Moreover, under the high-concurrency business scenario of microservices, our scheme can create less overhead due to latency.

\section{Committing rate}

In the three cases of low, moderate, and high contention, we evaluate the transactions' commit rate under our scheme. As shown in Fig. 10, 2PC* guarantees that the transactions are committed successfully even when the number of concurrent requests reaches a peak, while
2PC cannot be extended. When the transactions reached high con-tention, 2PC's commit rate dropped to almost zero-from 0.31 to 0.03 .

Under the scenario with 500 concurrent requests, we count the instances of each of the three states of the transaction, i.e., RUNNABLE, WAITING and BLOCKED. We then calculate their blocking rates. According to Fig. 11, the blocking rates of $2 \mathrm{PC}^{*}$ and $2 \mathrm{PC}$ both peaked at $450 \mathrm{~ms}$ and were $31 \%$ and $97 \%$, respectively; thus, $2 \mathrm{PC} *$ is more than 3 times better than $2 \mathrm{PC}$ in terms of the committing rate. At this point, almost all threads in $2 \mathrm{PC}$ were blocked, which is the key reason for its commit rate almost reaching zero. $2 \mathrm{PC}^{*}$ is also affected by the increase to high-level contention, although it is less sensitive than 2PC because it avoids aborting and retrying transactions. Compared with the traditional approach, $2 \mathrm{PC}^{*}$ improves the performance of transaction committing by 3 to 4.5 times.

\section{Transaction compensation}

We continuously request the CreateOrder interface in the weak network environment with 100 concurrent threads, for which the running time is $30 \mathrm{~s}$. As shown in the experimental data (Fig. 12), the transactions under $2 \mathrm{PC}^{*}$ and $2 \mathrm{PC}$ occurred with $23,853.6$ and 21,976.1 exceptions, respectively. We then recover the net-work environment and perform the transaction compensation process.

Our scheme can continuously compensate for exceptional transactions, which takes less than $3 \mathrm{~s}$ in total. $2 \mathrm{PC} *$ can be maintained at approximately 4831.4 groups per second, while $2 \mathrm{PC}$ has little ability to compensate. Based on BASE theory, $2 \mathrm{PC}^{*}$ uses the CMQ asynchronously to comp-ensate for these failed transaction steps. In other words, it improves the fault tolerance of the system, which is also essential in large distributed systems, such as microservices. 


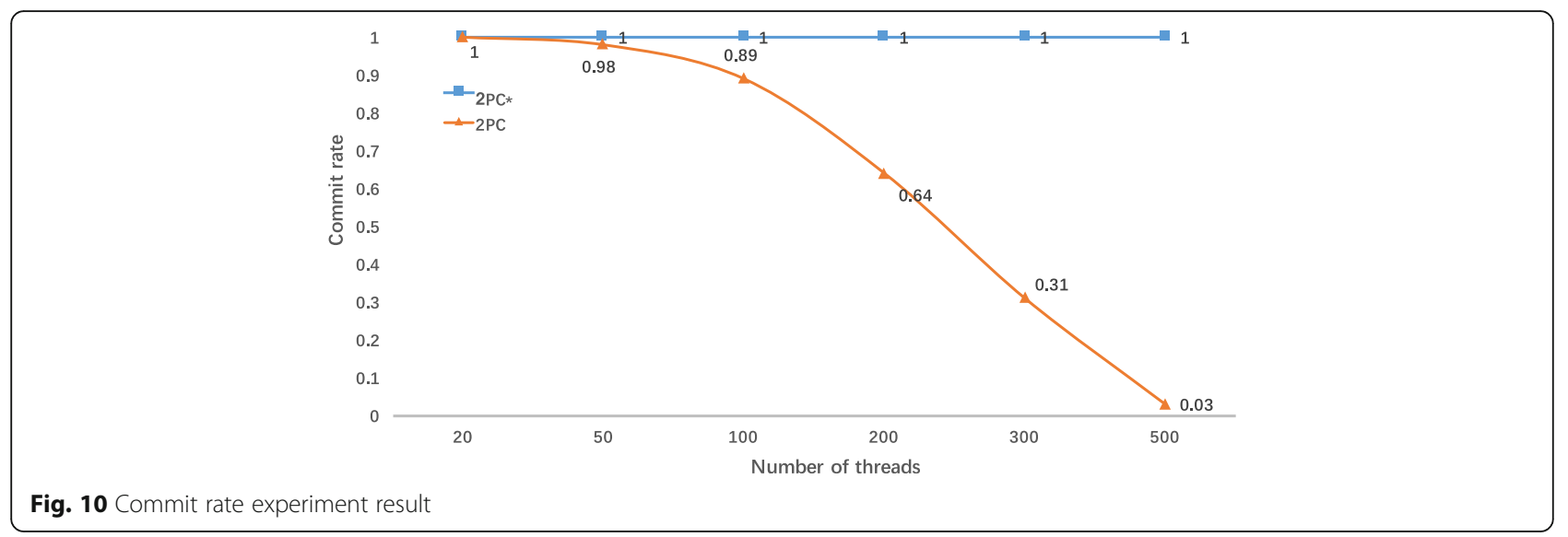

\section{Deployment and operations in cloud platform}

Finally, we deployed our middleware solution in Ctrip's intelligent PaaS (Platform as a Service) cloud platform [33], called CPaaS Fig. 13. Its core includes three basic modules, namely, the big data platform, microservice application platform and application integration platform. The microservice business is deployed in the microservice application platform. Mean-while, application performance monitoring is responsible for managing the monitoring of the service interfaces. More importantly, our transactional middleware is deployed in the application integration platform, which is responsible for the distributed transaction control of microservices deployed in the CPaaS platform.

Through the monitoring system of the CPAAS cloud plat-form, we obtained the performance data of our scheme over 3 months. The results are shown in Table 8 below. TPS can be maintained at $\sim 700$ new transactions/s with an RT of no more than 96 ms. Most importantly, the transaction commit rate is always $100 \%$, and transaction compensation can be successfully completed. Therefore, the long-term monitoring results prove that our scheme is stable and has universal app-licability in cloud computing.

\section{Related work}

In academia and industry, much of the recent work is still focused on transaction concurrency control using 2PC comb-ined with OCC in distributed databases, such as H-Store [32], VoltDB [34] by Michael and Samuel et al. In both $\mathrm{H}$-Store and VoltDB, where data is assigned to different partitions according to specific rules, one of the great contributions of $\mathrm{H}$-Store is that most transactions can be performed in a single partition, thus greatly reducing the additional overhead of concurrency control. For example, $\mathrm{H}$-Store can avoid the overhead of concurrent protocols with single-thread model for absolute single-partition transaction. It can supplement a few cross-partition transactions with the lightweight concur-rency protocol [35] to ensure possibility of serializability. However, as we have mentioned in this paper, these dist-ributed databases can only be applied to the single database and cannot be extended across

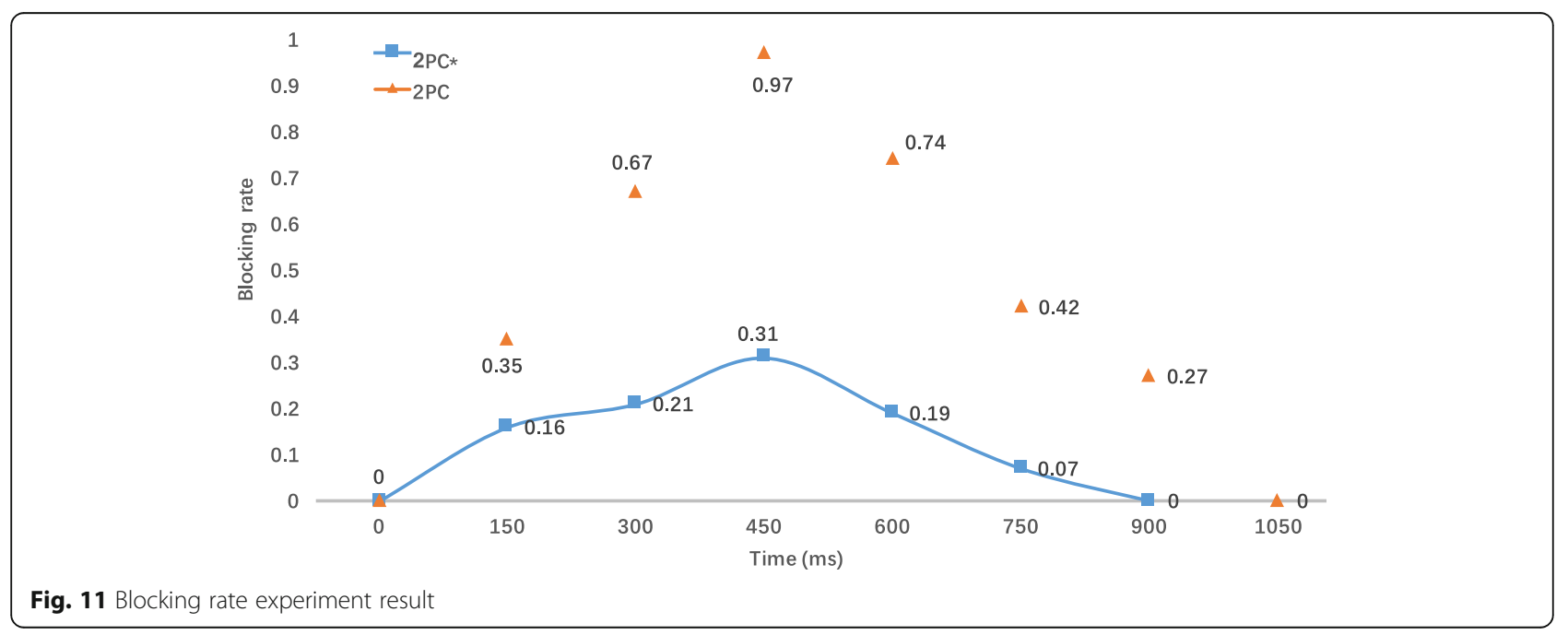




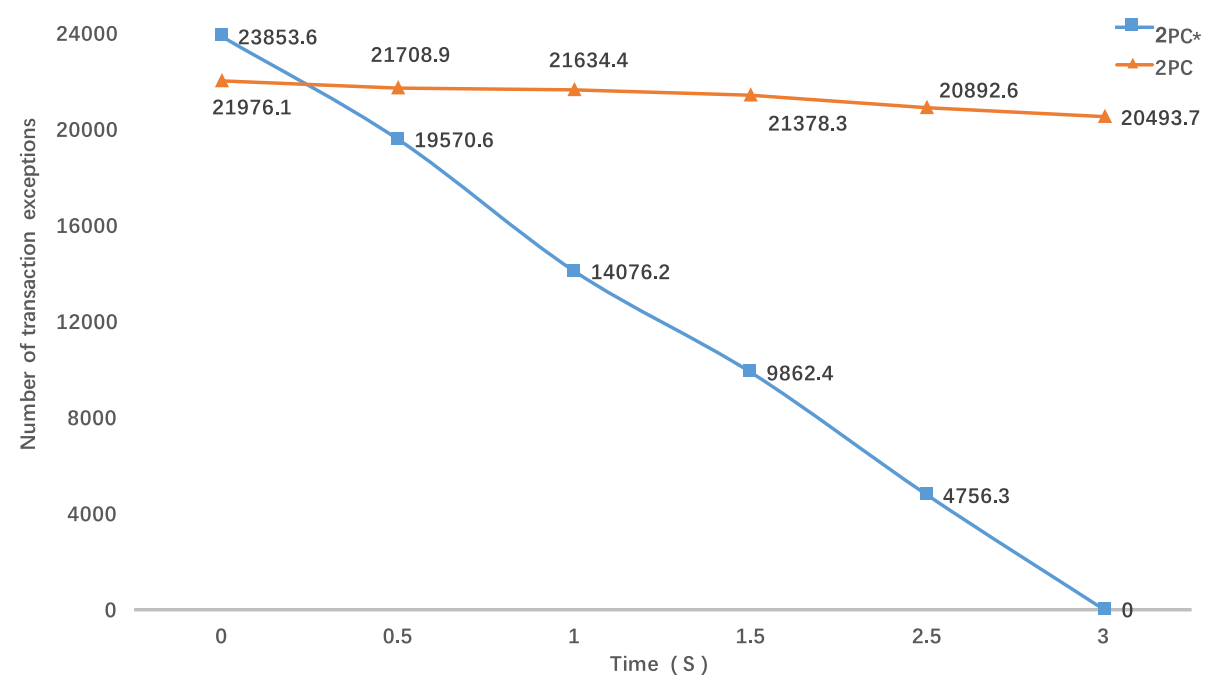

Fig. 12 Transaction compensation experiment result

multiple microservices, while $2 \mathrm{PC}^{*}$ improved this function.

The multi-data-center consistency (MDCC) proposed by Tim Kraska et al. [36] used a commit method based on optimistic control, which was frequently used for storage across data centers. Therefore, MDCC does not require a global master node or a static data partitioning approach, and provides additional overhead similar to the design of eventual consistency. MDCC is based on Generalized Paxos [37] design, combined with Commutative Operations support. Therefore, MDCC performs better than any synchronous commit method. The reason is that it requires only single message to commit most transactional requests between multiple data centers.

Google's proposed Percolator [2] adopts OCC to support Snapshot Isolation [38]. Percolator compensates for the lack of batch processing of document updates in systems such as MapReduce [39]. It supports incremental document processi-ng and has been deployed by Google in its internal web sear-ch system. To improve throughput, Percolator allows multip-le clients to fetch documents simultaneously, and to provide isolation between different clients, it uses $2 \mathrm{PC}$ combined with MVCC for transaction support. Percolator has improved the timeliness of Google web search results by $50 \%$ since Google deployed it. The SAOL locking mechanism in $2 \mathrm{PC}^{*}$ is also borrowed from the Percolator's design. SAOL uses the snapshot to breaking down locks in transactions into multiple levels of optimistic control, thereby reducing the blocking overhead of synchronous locks in a transaction.

In the Sinfonia [40] system built by Marcos K Aguilera et al., the concept of Min Transaction was innovatively

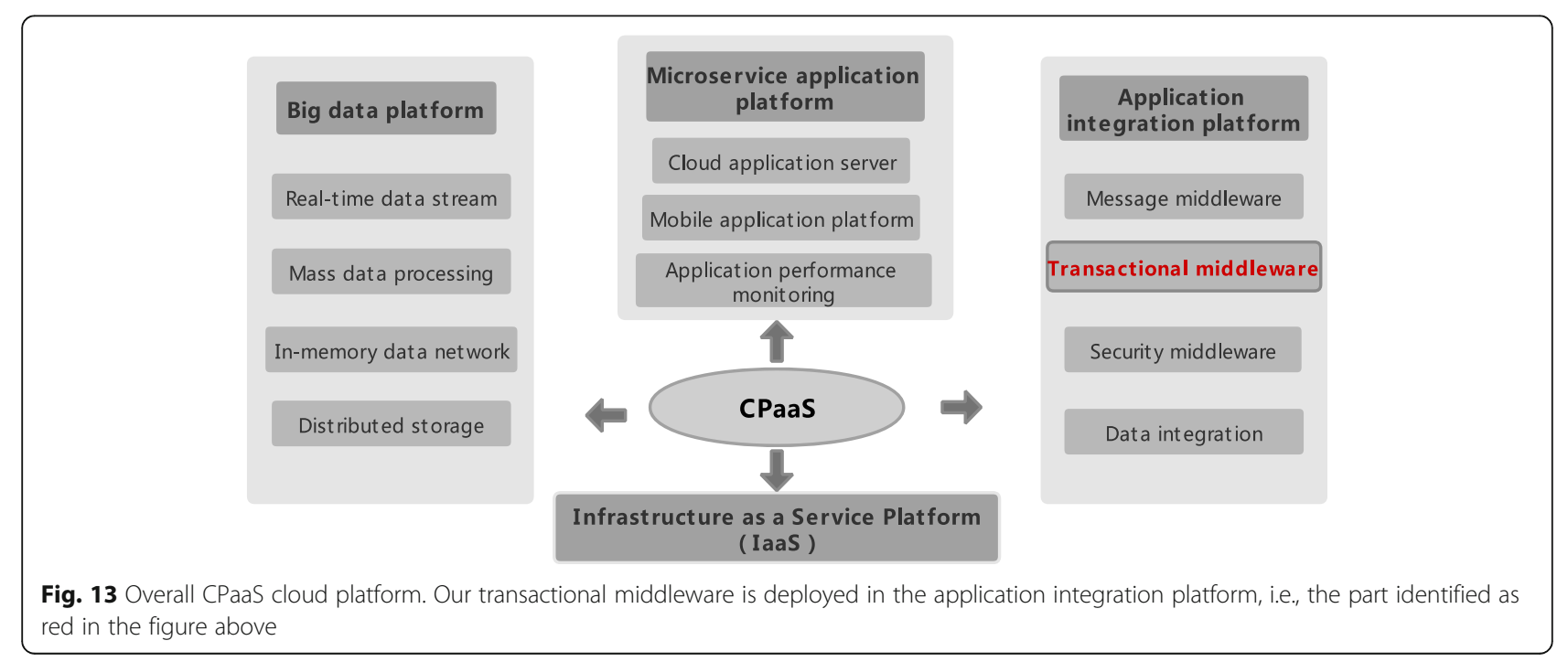


Table 8 Data consistency experiment data

\begin{tabular}{llllll}
\hline No. & Date & TPS & Commit Rate & $\begin{array}{l}\text { T } \\
(\mathrm{ms})\end{array}$ & Compensation \\
\hline $\mathbf{1}$ & August & 680.5 & 95.8 & $100 \%$ & 984 \\
$\mathbf{2}$ & September & 701.3 & 89.3 & $100 \%$ & 879 \\
$\mathbf{3}$ & October & 692.7 & 98.5 & $100 \%$ & 1034 \\
\hline
\end{tabular}

propos-ed, and its transaction implementation was also based on the 2PC protocol. Min Transaction is performed by locking the access object in the first phase of the Transaction and then committing it in the second phase. Sinfonia perfected the $2 \mathrm{PC}$ mechanism and came up with the concept of $1 \mathrm{PC}$ so that a single message exchange could commit the entire transaction for the coordinator. Unfortunately, the $1 \mathrm{PC}$ protocol of the Sinfonia is not able to be scaled for multiple microservices, as microservices often exist in the form of crossservices and cross-resources, which is the reason that $2 \mathrm{PC}$ * is still based on the two-phase commit protocol.

Andrei Furda et al. migrated microservices into the cloud computing environment and addressed several key issues in the process, one of which was data consistency [41]. That is, the challenges encountered in migrating legacy code runs can be summarized as operating decentralized data repositories from a centralized data repository to the microservices. Guy Pardon et al. studied the BAC theorem (backup, availability, and/or consistency) [42], which is an effective solution for the consistent disaster recovery for microservices, and is inspired by the CAP theorem. We also improved the fault tolerance of the $2 \mathrm{PC}^{*}$ protocol, which is similar to the even-tual consistency they proposed, except that we borrowed from the BASE theory.

Zhang et al. proposed GRIT's distributed transaction model across microservices [3], which utilizes deterministic database technology and OCC to process data consistently. During the execution phase, transactions are optimally exec-uted by capturing their read and write operations. Then, at commit time, this method performs a conflict check and makes a global commit decision. Logically committed trans-actions are first transferred to the $\log$ and then executed asyn-chronously to carry out the database business. GRIT works at the procedural language level of the different data-bases, and $2 \mathrm{PC}^{*}$ also adopts OCC's optimistic control, but we focus on transaction concurrency optimization and data consistency constraints.

\section{Conclusion}

This paper proposed 2PC*, a novel concurrency control protocol for distributed transactions in multimicroservice modules. For this purpose, we designed a novel secondary asynchronous optimistic lock, which can avoid the locks that are held in the transaction process. 2PC* utilizes a novel transaction concurrency control protocol, which is able to reduce the probability of concurrent conflicts among multiple transactions. Compared to the original $2 \mathrm{PC}, 2 \mathrm{PC}$ can extract greater concurrency across multiple microservices. Finally, we implemented a middleware prototype based on $2 \mathrm{PC}^{*}$ and applied it to a case of Ctrip MSECP deployed in the $\mathrm{CPaaS}$ cloud platform. The experimental results demonstrate that in high-level contention scenarios, our scheme has a higher throughput and lower latency than 2PC. Additionally, through long-term application performance monitoring by the CPaaS cloud platform, our scheme effectively supports distributed transaction concurrency control in a multi-microservice system.

In addition, we intend to continue some of our research in future work. We will adapt our scheme to some microservice frameworks in addition to Spring Cloud and Dubbo, which were discussed in this paper. As cloud computing becomes more popular, we can deploy it in DevOps [43, 44] in the PaaS [33, 45] cloud platform. In the IoT (Internet of Things) [27, 29], where cloud computing takes place, our scheme can also be extended. We also need to improve the QoS (quality of service) $[29,46]$ of microservices under various scenarios for mobile social networks [47] and hybrid networks [48] in the future.

\section{Abbreviations}

2PC: Two-phase commit; OCC: Optimistic concurrency control; MVCC: Multiversion concurrency control; TCM: Transaction coordination manager; RPC: Remote procedure call

\section{Acknowledgements}

Our deepest gratitude goes to the anonymous reviewers for their valuable suggestions to improve this paper.

\footnotetext{
Authors' contributions

Pan Fan designed and developed the proposed protocol as well as implemented the middleware solution based on cloud computing platform. Jing Liu directed working research and paper writing, and gave the experimental scheme. Wei Yin and Hui Wang provided experimental environments and designed use cases. Xiaohong Chen and Haiying Sun gave guidance on the grammar and abstract of this paper. The authors read and approved the final manuscript.

\section{Authors' information}

Pan Fan is studying for a master's degree in engineering at East China Normal University, China. His research interests include distributed computing, cloud computing, and formal method.

Jing Liu is currently a professor of computer science with East China Normal University, China. In recent years, she has been involved in the area of
} 
model-driven architecture. She currently focuses on the design of real-time embedded systems and cyber-physical systems.

Wei Yin graduated from Northwestern Polytechinical University, China, he is currently a Senior Engineer of SAVIC Ltd. In recent years, he is involved in the area of Avioncs design, Software Engineering, System Engineering etc. His current work focuses on the design of the software and airworthness of Avionics.

Hui Wang received a master's degree in Nanjing University of Aeronautics and Astronautics, China. He is currently a researcher of SAVIC Ltd. His research interests include software verification and formal method. Xiaohong Chen received a Ph.D. degree in Chinese Academy of Sciences, China, she is currently working as a master's tutor at East China Normal University, China. Her research interests include formal method, cyberphysical systems and artificial intelligence.

Haiying Sun received a Ph.D. degree in East China Normal University, China, her research interests include formal method, system simulation and modeldriven engineering.

\section{Funding}

This paper is partially supported by funding under National Key Research and Development Project 2017YFB1001800, NSFC Project 61972150, and Shanghai Knowledge Service Platform Project ZF1213.

\section{Availability of data and materials}

The full experimental data and the description of the experiment setup are provided in this manuscript in section Evaluation and our github, https:// github.com/Leofan93/2pc-star.

\section{Competing interests}

The authors declare that they have no competing interests.

\section{Author details}

${ }^{1}$ East China Normal University, Shanghai 200062, China. ${ }^{2}$ Shanghai Avionics co. Ltd, Shanghai, China.

Received: 30 January 2020 Accepted: 23 June 2020

Published online: 23 July 2020

\section{References}

1. Larrucea X, Santamaria I, Colomo-Palacios R, Ebert C (2018) Microservices. IEEE Softw 35(3):96-100. https://doi.org/10.1109/MS.2018.2141030

2. Corbett JC, Dean J, Epstein M, Fikes A, Frost C, Furman J, Ghemawat S, Gubarev A, Heiser C, Hochs-child P et al (2013) Spanner: googles globally distributed data-base. ACM Transact Comput Syst (TOCS) 31(3):8. https://doi. org/10.1145/2491245

3. Zhang G, Ren K, Ahn JS et al (2019) GRIT: consistent distributed transactions across polyglot microservices with multiple databases[C]. In: 2019 IEEE 35th international conference on data engineering (ICDE). IEEE. https://doi.org/10, 1109/ICDE.2019.00230

4. Mohan C, Lindsay B, Obermarck R (1986) Transaction management in the $R^{*}$ distributed database management system. ACM Trans Database Syst (TODS) 11(4):378-396

5. Thomson A, Diamond T, Weng SC et al (2012) Calvin: fast distributed transactions for partitioned database systems [C]. In: Acm Sigmod international conference on management of data. ACM. https://doi.org/10. $1145 / 2213836.2213838$

6. Hwang E, Kim S, Yoo TK et al (2015) Resource allocation policies for loosely coupled applications in heterogeneous computing systems[]]. IEEE Trans Parallel Distributed Syst:1-1. https://doi.org/10.1109/TPDS.2015.2461154

7. Du J, Sciascia D, Elnikety S, Zwaenepoel W, Pedone F (2014) Clock-RSM: low-latency inter-datacenter state machine replication using loosely synchronized physical clocks. 2014 44th annual IEEE/IFIP international conference on dependable systems and networks, Atlanta, pp 343-354. https://doi.org/10.1109/DSN.2014.42

8. YugaByte. https://www.yugabyte.com/. Accessed 10 May 2019.

9. FoundationDB. https://www.cockroachlabs.com/. Accessed date 10 May 2019.

10. Zhang S, Zhu S (2013) Server structure based on netty framework for internet-based laboratory [C]. In: Control and automation (ICCA), 2013 10th IEEE international conference on. IEEE. https://doi.org/10.1109/ICCA.2013. 6564990
11. Bershad BN, Anderson TE, Lazowska ED, Levy HM Lightweight remote procedure call. ACM Trans Comput Syst 8(1):37-55. https://doi.org/10.1145/ 74850.74861

12. He S, Zhao L, Pan M (2018) The Design of Inland River Ship Microservice Information System Based on spring cloud [C]. In: 5th international conference on information science and control Engin-eering (ICISCE). https://doi.org/10.1109/ICISCE.2018.00120

13. Dubbo. http://dubbo.apache.org/. Accessed 9 Mar 2019.

14. Herlihy MP, Wing JM (1990) Linearizability: a correctness condition for concurrent objects. ACM Transact Program Lang Syst (TOPLAS) 12(3):463-492

15. Herlihy M Apologizing versus asking permission: optimistic concurrency control for abstract data types [J]. ACM Trans Database Syst 15(1):96-124. https://doi.org/10.1145/77643.77647

16. Nishi T, Yushin et al (2017) An efficient deadlock prevention policy for noncyclic scheduling of multicluster tools []]. In: IEEE transactions on automation science and engineering. https:/doi.org/10.1109/TASE.2017.2771751

17. Breitbart Y, Garcia-Molina H, Silberschatz A (1992) Overview of multidatabase transaction management. VLDB J 1:181-239. https://doi.org/ 10.1007/BF01231700

18. Ardekani MS, Sutra P, Shapiro M (2013) Non-monotonic snapshot isolation: scalable and strong consistency for geo-replicated transactional systems[C]. https://doi.org/10.1109/SRDS.2013.25

19. Attiya $\mathrm{H}$, Ellen F, Morrison A Limitations of highly-available eventuallyconsistent data stores [J]. In: IEEE transactions on parallel and distributed systems, p 1. https://doi.org/10.1109/TPDS.2016.2556669

20. Luo C, Okamura H, Dohi T (2013) Modeling and analysis of multi-version concurrent control [C]. In: 2013 IEEE 37th annual computer software and applications conference. IEEE. https://doi.org/10.1109/COMPSAC.2013.11

21. snowflake. https://github.com/twitterarchive/snowflake. Accessed 11 June 2019.

22. Tarjan (2008) Depth-first search and linear graph algorithms [C]. In: Symposium on Switching \& Automata Theory. IEEE. https:/doi.org/10.1109/SWAT.1971.10

23. Chaudhuri K, Doligez D, Lamport L et al (2010) The TLA+ proof system: building a heterogeneous verification platform $[\mathrm{M}] / /$ theoretical aspects of computing - ICTAC 2010. Springer, Berlin Heidelberg. https://doi.org/10. 1007/978-3-642-14808-8_3

24. Github. https://github.com/Leofan93/2pc-star. Accessed 11 June 2019.

25. Taibi, Toufik Formal specification and validation of multi-agent behaviour using tla+ and tlc model checker. Int J Artificial Intel Soft Comput 1(1):99. https://doi.org/10.1504/ijaisc.2008.021266

26. Huang $X$, Zhang $Y$, Xing $C$ et al (2012) Paxos-based memory data replication in stock trading system [C]. In: IEEE computer software \& applications conference. IEEE Computer Society. https://doi.org/10.1 109/COMPSAC.2012.46

27. CompletableFuture. https:/docs.oracle.com/javase/8/docs/api/java/util/ concurrent/CompletableFuture.html. Accessed 11 June 2019.

28. PlatformTransactionManager. https://docs.spring.io/spring/docs/5.1.3. RELEASE/spring-framework-reference/data-access.html\#transactionstrategies. Accessed 11 June 2019.

29. ReentrantLock. https://docs.oracle.com/javase/7/docs/api/java/util/ concurrent/locks/ReentrantLock.html. Accessed 11 June 2019.

30. Artho C, Gros Q, Rousset G et al (2017) Model-based API testing of apache ZooKeeper [C]. In: 2017 IEEE international conference on software testing, verification and validation (ICST). IEEE. https://doi.org/10.1109/ICST.2017.33

31. Ngnix. https://www.nginx.com/resources/wiki/. Accessed 15 June 2019

32. Eureka. https://spring.io/projects/spring-cloud-netflix. Accessed 15 June 2019.

33. Yin Y, Lu C, Xu Y, Wan J, Zhang H, Mai Z (2019) QoS prediction for service recommendation with deep feature learning in edge computing environment. In: Mobile networks and applications. https://doi.org/10.1007/ s11036-019-01241-7

34. Bernstein D (2014) Today's tidbit. VoltDB [J] 1(1):90-92. https://doi.org/10. 1109/MCC.2014.25

35. Pedreira P, Lu Y, Pershin S (2018) Rethinking concurrency control for inmemory OLAP DBMSs. In: IEEE 34th international conference on data engineering (ICDE). https://doi.org/10.1109/ICDE.2018.00164

36. Kraska T, Pang G, Franklin M, Madden S (2012) MDCC: multi-data center consistency. In: Proceedings of the 8th ACM European conference on computer systems, EuroSys 2013. https://doi.org/10.1145/2465351.2465363

37. Pires M, Ravi S, Rodrigues R (2017) Generalized Paxos made byzantine (and less complex) [C]. In: International symposium on stabilization, safety, and security of distributed systems. https:/doi.org/10.1007/978-3-319-69084-1_14 
38. Wei H, Huang Y, Lu J (2017) Parameterized and runtime-tunable snapshot isolation in distributed transactional key-value stores [C]. In: 2017 IEEE 36th symposium on reliable distributed systems (SRDS). IEEE. https://doi.org/10. 1109/SRDS.2017.11

39. Xu X, Tang M (2017) A new approach to the cloud-based heterogeneous MapReduce placement problem [J]. IEEE Trans Serv Comput 9(6):862-871. https://doi.org/10.1109/TSC.2015.2433914

40. Aguilera MK, Merchant A, Shah MA, Veitch AC, Karamanolis CT (2009) Sinfonia: a new paradigm for building scalable distributed systems. ACM Trans Comput Syst 27(3). https://doi.org/10.1145/1294261.1294278

41. Furda CF, Zimmermann O, Kelly W, Barros A (2018) Migrating Enterprise legacy source code to microservices: on multitenancy, Statefulness, and data consistency. IEEE Softw 35(3):63-72. https://doi.org/10.1109/MS.2017. 440134612

42. Pardon G, Pautasso C, Zimmermann O (2018) Consistent disaster recovery for microservices: the BAC theorem. IEEE Cloud Comput 5(1):49-59. https:// doi.org/10.1109/MCC.2018.011791714

43. Gao H, Duan Y, Shao L, Sun X (2019) Transformation-based processing of typed resources for multimedia sources in the loT environment. Wirel Netw. https://doi.org/10.1007/s11276-019-02200-6

44. Gao H, Xu Y, Yin Y, Zhang W, Li R, Wang X (2019) Context-aware QoS prediction with neural collaborative filtering for internet-of-things services. IEEE Internet Things J. https://doi.org/10.1109/JIOT.2019.2956827

45. Ebert C, Gallardo G, Hernantes J et al (2016) DevOps [J]. IEEE Softw 33(3):94100. https://doi.org/10.1109/MS.2016.68

46. Bernstein D (2014) Cloud foundry aims to become the OpenStack of PaaS. Cloud Comput IEEE 1(2):57-60. https://doi.org/10.1109/MCC.2014.32

47. Yin Y, Xia J, Yu L, Xu Y, Xu W, Yu L (2019) Group-wise itinerary planning in temporary Mobile social network. IEEE Access 7:83682-83693. https://doi. org/10.1109/ACCESS.2019.2923459

48. Gao H, Liu C, Li Y, Yang X (2020) V2VR: reliable hybrid-network-oriented V2V data transmission and routing considering RSUs and connectivity probability. In: IEEE transactions on intelligent transportation systems(T-ITS). https://doi.org/10.1109/TITS.2020.2983835

\section{Publisher's Note}

Springer Nature remains neutral with regard to jurisdictional claims in published maps and institutional affiliations.

\section{Submit your manuscript to a SpringerOpen ${ }^{\circ}$ journal and benefit from:}

- Convenient online submission

- Rigorous peer review

- Open access: articles freely available online

- High visibility within the field

- Retaining the copyright to your article

Submit your next manuscript at $\boldsymbol{\nabla}$ springeropen.com 